\title{
A Mixed-fidelity Numerical Study for Fan-distortion Interaction
}

\author{
Yunfei Ma \\ PhD candidate \\ Department of Engineering \\ University of Cambridge \\ United Kingdom, CB2 1PZ \\ Email:ym324@cam.ac.uk \\ Jiahuan Cui * \\ Assistant Professor \\ School of Aeronautics and Astronautics \\ ZJU-UIUC Institute \\ Zhejiang University \\ People's Republic of China, 310007 \\ Email: jiahuancui@intl.zju.edu.cn

\begin{abstract}
Nagabhushana Rao Vadlamani
Senior Research Associate

Department of Engineering

University of Cambridge

United Kingdom, CB2 1PZ

Email:nrv24@cam.ac.uk
\end{abstract} \\ Paul Tucker \\ Professor \\ Department of Engineering \\ University of Cambridge \\ United Kingdom, CB2 1PZ \\ Email:pgt23@cam.ac.uk
}

\section{Abstract}

Inlet distortion often occurs under off-design conditions when a flow separates within an intake and this unsteady phenomenon can seriously impact fan performance. Fan-distortion interac-

*Address all issues to this author. 
tion is a highly unsteady aerodynamic process into which high-fidelity simulations can provide detailed insights. However, due to limitations on the computational resource, the use of an eddy resolving method for a fully resolved fan calculation is currently infeasible within industry. To solve this problem, a mixed-fidelity CFD method is proposed. This method uses the Large Eddy Simulation (LES) approach to resolve the turbulence associated with separation, and the Immersed Boundary Method with Smeared Geometry (IBMSG) to model the fan. The method is validated by providing comparisons against the experiment on the Darmstadt Rotor, which shows a good agreement in terms of total pressure distributions.

A detailed investigation is then conducted for a subsonic rotor with an annular beamgenerating inlet distortion. A number of studies are performed in order to investigate the fans influence on the distortions. A comparison to the case without a fan shows that the fan has a significant effect in reducing distortions. Three fan locations are examined which reveal that the fan nearer to the inlet tends to have a higher pressure recovery. Three beams with different heights are also tested to generate various degrees of distortion. The results indicate that the fan can suppress the distortions and that the recovery effect is proportional to the degree of inlet distortion. 


\section{Introduction}

Inlet distortion often occurs at off-design points when flows separate within an intake. This unsteady phenomenon can seriously impact fan performance. The current trend of engine design is to make the intakes shorter and as a consequence, an increased interaction between the inlet distortions and a downstream fan may further deteriorate the performance. The fan-distortion interaction is a highly unsteady aerodynamic phenomenon that needs to be investigated using high-fidelity methods. This is because conventional steady or unsteady Reynolds-averaged Navier-Stokes (RANS) modelling cannot resolve turbulence, although it is sometimes feasible for applications in industry [1]. Due to the limitations of turbulence models, results may vary significantly with different RANS models [2-5] when predicting highly separated flows. Eddy resolving simulation techniques such as DNS/LES and hybrid LES/RANS have been shown to yield more promising results [6-8]. However, the cost of such simulations can be tremendous (Gourdain [9]).

To enable such simulations however, a high-fidelity method can be jointly used with lowfidelity methods, an approach known as 'mixed fidelity modelling'. For example, when simulating the separated flow of an intake, one may use a lower-order model for the blades instead of resolving the blades as a 'boundary condition' for the separation region. This is feasible because pursuing computational accuracy does not necessarily correspond to a higher fidelity. As long as the calibrated low-order model is capable of achieving this target within an acceptable tolerance, it can in fact partially replace higher-fidelity methods. According to a recent report by NASA [10], "coupled problems, such as separation, transition, hear transfer, aeroacoustics, aeroelastics, etc., desire a physics-based, predictive modelling approach that must capture the underlying physics more accurately than ever before". Hence, for these coupled problems, mixed-fidelity modelling could achieve the target at a lower cost.

The low-fidelity method we mention here essentially concerns geometrical modelling, which also has various types, as shown in Figure 1. The lowest level, the Immersed Boundary Method with Smeared Geometry (IBMSG) approach, also known as the blade body force approach, assumes that the number of blades is infinite, and that the flow is circumferentially averaged. The inviscid IBMSG, firstly proposed by Marble [11] simply uses the force normal 
to the blade surfaces to model the flow turning effects. Viscous body force models were introduced by Xu [12] who used such models for unsteady flow calculations and for the investigation of distortion problems. This model assumes a number of force-velocity relations to model loss effects. In the middle of this hierarchy, the Immersed Boundary Method is used for real geometries. This model was proposed by Peskin [13] and was applied by Fadlun [14] for some simple geometrical calculations. Defoe [15] used this method to model individual rotor blades in order to predict the intake noise with inlet distortion. The present research is aimed at providing a mixed-fidelity CFD method using the IBM for the inlet distortion generator and the IBMSG approach for the rotational fan. With such methods, the emphasis will be on the influence of the fan on intake distortion.

To date, much numerical and experimental research [16-18] has been conducted to simulate intake distortion. Various distortion generators were designed to reproduce similar flow conditions to those in the laboratory. Among these studies, Wartzek [19] investigated distortion patterns using a transonic compressor stage. In his experiment, both circumferential and whole spanwise intake distortions were investigated, providing essential results for numerical validation. Some RANS and URANS simulations were conducted also by Fidalgo [20], Barthmes et al. [21], etc., although research on intake-fan interaction within a high-fidelity simulation is rare. Here, we apply the mixed-fidelity method to study some key factors for the fan-intake interaction. Based on this method, Ma et al. [22] found that a downstream fan can significantly reduce the separation bubble, and hence the reattachment point can be much earlier. The mechanism revealed in their research shows that the fan influences intake separation mainly via main flow. Hence, the present research is to delve into some key factors, such as fan location, distortion size, etc., which may affect the reduction of inlet distortion.

The goal of this research is to enable the simulation of unsteady, complex flows whilst maintaining accuracy. This involves exploring a mixed-fidelity modelling approach that applies the IBMSG model for the fan and an eddy resolving method for the separated flows. The research consists of two parts. Firstly, the method is validated on a transonic rotor (Darmstadt Rotor). The performance map and total pressure distributions are also compared against experimental results. To investigate how the fan impacts the inlet distortion, different fan locations and de- 
grees of distortion are explored on a Darmstadt-derived rotor. Such a parametric study focuses on the key factors that can offer insights into the interaction between fan and inlet distortion.

\section{Numerical Method}

\subsection{IBM for the distortion generator}

To reduce the cost of high-fidelity modelling without sacrificing accuracy, the Immersed Boundary Method (IBM) provides a potential means to model the components, such as the rotor and stator blades in engines. By using this approach, the mesh can be significantly simplified. Essentially, the idea of IBM is to replace boundaries with forces which can be either a point or body force. This idea was first proposed by Sirovich [23], who applied it to solve linearised initial and boundary problems. In this work, he regarded the boundary surfaces as a distribution of sources in the flow which were then directly included in the Navier-Stokes equations. Consequently, the boundary problems were transformed into standard PDEs with source terms. This was further demonstrated by Sirovich [24] analytically, such that the transformation was essentially a solution operator acting on the boundary data. Following this work, Salathé [25] extended this method to the study of compressible hydrodynamics, after these which, Peskin [13] completed a strict mathematical justification of the Immersed Boundary Method and applied it to the study of fluid-structure interaction. Hence, this method is applicable to the study of engine components, such as various types of distortion generators. The force then can be modelled according to Goldstein et al. [26] as,

$$
\begin{array}{r}
f(\boldsymbol{x}, t)=\alpha \int_{0}^{t} \Delta u d t+\beta \Delta \boldsymbol{u}, \\
\Delta \boldsymbol{u}=\boldsymbol{u}\left(\boldsymbol{x}_{0}, t\right)-\boldsymbol{u}_{0}\left(\boldsymbol{x}_{0}, t\right) .
\end{array}
$$

Here, the subscript 0 represents the solid boundary and the coefficients $\alpha$ and $\beta$ are negative constants. This force is evaluated as a function of the difference between the actual velocity and designed one, which can be also regarded as a proportional-integral (PI) feedback controller. Peskin [13] demonstrated that the value of the force is independent of the two coefficients $\alpha$ and $\beta$, once the solution has converged. To ensure convergence, the two coef- 
ficients should be carefully selected. They are associated with two important parameters: the frequency of the integral part of the feedback forcing: $\frac{1}{2 \pi} \sqrt{|\alpha|}$ and the damping factor of the proportional part of the feedback forcing: $-\frac{\beta}{2 \sqrt{|\alpha|}}$. Hence, $\alpha$ must be large enough to ensure that the frequency $\frac{1}{2 \pi} \sqrt{|\alpha|}$ is much bigger than any other frequencies in the flow [14]. This means that the feedback force should change as rapidly as possible and direct the flow in the desired direction. Also, the time step should be chosen to ensure numerical stability:

$$
\Delta t<\frac{-\beta-\sqrt{\beta^{2}-2 \alpha k}}{\alpha}
$$

where the order of $k$ is 1 .

\subsection{IBMSG for the fan}

The fan is modelled using IBM for 'Smeared Geometries' (IBMSG) which approach assumes that a rotating fan can be regarded as a set of an infinite number of infinitesimally thin blades. The forces are circumferentially averaged in every cell in the blade region in order to turn the flow circumferentially in the desired direction. Cao et al. [27] used this method to study intake separation under high angle of incidence and demonstrated the capability of this method in capturing key flow features. The normal force in this model is still controlled by Equation 1 and the parallel force is modelled by a typical force-velocity relation. This relation was specifically investigated by $\mathrm{Xu}$ [12]. He applied this modelled viscous force to study three-dimensional unsteady flows with low-order distortion using a viscous body force that was considered to be proportional to $\rho \boldsymbol{u}^{2}$. The coefficient for this proportional relation is constant and can be approximated from fine mesh RANS. It was found that this simple relation performed well when characterising the unsteady behaviour caused by blade-row interaction. Compared to eddy resolving methods, the computational time required can be reduced by two orders of magnitude. Following this work, Cao [28] and Watson [29] further developed a viscous body force modelling technique which worked well in the study of intake distortion. In this 
case, the parallel force is given as,

$$
\boldsymbol{f}_{p}=-K(r) \rho \boldsymbol{u}_{r e l}^{2}, K(r)=4 k_{1} s^{2}+k_{1},
$$

where $s$ is the fraction of span and the coefficient $k_{1}$ is a calibration constant set to 0.2 (This value is obtained by calibrating the characteristic map against the experimental and/or resolved blade data at the design speed. More details can be found in Reference [29].) The finite blade thickness introduces blockage to the flow passing through the passage. This effect is modelled as,

$$
\lambda=1-\frac{1}{2} \frac{\left(t_{1}+t_{2}\right)}{S(r) \cos \beta},
$$

where $S(r)$ is the surface pitch and $t_{1}+t_{2}$ is the total blade thickness (which is based on the intersection of the normal to the camber line of the blade. Details can be found in [28]). Both the normal and parallel forces are added to the momentum equations of the compressible Navier-Stokes equations.

Although the model cannot provide very much detail within the blade region, it is still able to provide a 'boundary' for some target region. This is the core idea of 'mixed-fidelity modelling'. For the present research concerning fan-intake interaction, the focus is on the separation domain, which will be significantly influenced by both the intake and the downstream rotor. As long as the modelled components around this domain of focus domain can replicate a number of important characteristics, this method can be useful for high-fidelity modelling.

\subsection{Numerical Framework}

Two test cases are used relating to validation and investigation, respectively. The validation is conducted on a transonic rotor, named the Darmstadt Rotor [16-18]. In these studies, distortion generators were designed to reproduce the flow conditions in a real engine within the laboratory. Measurements by Lieser [16] and Bitter [30] show that the compressor perfor- 
mance is most sensitive to the distortions encountered at the tip. Hence, a periodic distortion generator is placed upstream of the tip of the fan in order to reproduce the distortion encountered over the intake lip at high angles of attack. The present numerical simulation includes an IBM-modelled distortion generator, which covers $120^{\circ}$ in circumferential direction and an IBMSG-modelled rotor and stator, as shown in Figure 2. The rest of the domain is computed using RANS. International Standard Metric Conditions ( $p_{0}=101325 \mathrm{~Pa}$ and $T_{0}=288.15 \mathrm{~K}$ ) are applied at the inlet flow and the target mass flow rate is $16.0 \mathrm{~kg} / \mathrm{s}$. This mass flow rate corresponds to the peak efficiency point at $100 \%$ rotational speed $(2094.39 \mathrm{rad} / \mathrm{s})$. The radial equilibrium boundary condition is used for the outlet. The key parameters of this rotor are listed in Table 1 and further details of relevant parameters can be found in [19]. The influence of the fan on inlet distortion is investigated using a simplified test case. Figure 3 illustrates the computational domain and the boundary conditions considered in this simplified setup, motivated by the experimental studies on the Darmstadt Rotor, albeit under $65 \%$ rotational speed $(1361.31 \mathrm{rad} / \mathrm{s})$ and $10.6 \mathrm{~kg} / \mathrm{s}$ mass flow rate. This is because the Darmstadt Rotor is a transonic rotor and this shock waves may exist in the duct at the design point. To simplify the problem, we need to avoid the influence of these shock waves. In addition, this test case employs the original duct and the rotating fan, with a periodic beam installed upstream of the fan. It features a sector duct with a distortion generator ('beam') of height ' $H$ ' and length ' $1.5 H$ ' placed at an axial distance of ' $12.5 H$ ' from the inlet. The fan is positioned at a streamwise distance of ' $5.25 \mathrm{H}$ from the beam and the velocity is normalised by $u_{\infty}$, measured in the main flow near the tip of the beam, corresponding to the outer edge velocity of the separated shear layer.

The primary objective of the current study is to capture the distortion generated on the lower wall. Hence, an inviscid boundary condition is imposed on the upper wall, which ensures that the pressure distribution due to the spinner is well represented at a reduced computational cost. A radial equilibrium boundary condition is imposed at the outflow and periodicity is imposed in the circumferential direction. The extent of the sector (corresponding to $5 H$ ) is sufficient to ensure that structures are decorrelated in the circumferential direction. In this mixed-fidelity framework, the beam is represented using a conventional IBM. The separated 
flow downstream of the beam is captured using an eddy resolving approach (LES), while the force field of the rotating fan is replicated using the IBMSG approach.

\section{COMPUTATION SETUP}

In this section, some preparatory work relating to the intake-fan interaction using mixedfidelity modelling is described. This includes the verification of the geometry simplification as well as grid independence studies. The former is performed using RANS, whereas the latter applies different sizes of mesh using LES.

\subsection{Geometry simplification}

To reduce the computational cost, we ignore the circumferential influence and extract a sector of the duct $\left(30^{\circ}\right)$ with periodic boundary conditions. Hence, the first thing to establish is that the periodic distortion generator does not significantly affect the axial and radial distribution of the separation. This is achieved by considering two cases, one with a $360^{\circ}$ beam and the other with a $120^{\circ}$ beam, respectively.

In Figure 4, inflectional points of the separation bubble are identified via the inflectional points of the velocity. The height in the middle of the separation bubble for the $360^{\circ}$ beam is much higher than that for the $120^{\circ}$ beam. However, the separation lengths are almost the same. This means that the symmetry only thickens the bubble. According to this feature, we may conclude that a symmetric beam (essentially a $360^{\circ}$ beam) can replace an asymmetric one in a meaningful way. In addition, although it is not possible to represent the bubble height, the trend of the bubble height under various different circumstances can still be investigated as long as the same original conditions are employed.

\subsection{Mesh quality}

This section examines the effect of the mesh on the intake distortions. Three sets of meshes were tested, and the number of nodes for the coarse, mid-size and fine grids were set to 1.1 million, 8.6 million and 60 million, as shown in Table 2. These differences represent the dimensionless spacing in the streamwise and spanwise directions.

Figure 5 shows the results of the LESs with the fan in terms of the mass flux and pressure. 
These distributions are extracted at $x=4.5 \mathrm{H}$. The figures indicate that the size of the grid does not have a visible influence on the mass flux and total pressure distributions. This is perhaps due to the fact that there is no typical boundary layer in the recirculation region, and hence the usual wall spacing can be relaxed. This indicates that the coarse grid is sufficient for the intake-fan interaction insofar as its effects on separation bubbles are considered.

\section{Validation}

\subsection{Darmstadt Rotor}

To verify the IBM- and IBMSG-modelled components are modelled accurately, the performance map was simulated using RANS with IBM and compared with the experiment from Wartzek [19].

In Figure 6, it is clear that the IBM beam and IBMSG-modelled fan can characterise the general trend in the performance. The pressure ratios for both cases are well captured. However, the near-stall point is not accurately predicted and the choking mass flow rate of 16.4 $\mathrm{kg} / \mathrm{s}$ cannot be approached, representing possible limitations of this method.

The total pressure distribution is also compared in order to investigate the response of the rotor and stator. To achieve the best performance, the design point at $16.0 \mathrm{~kg} / \mathrm{s}, 100 \%$ rotational speed is selected. Figure 7 shows the flow field of the Darmstadt Rotor with an IBM beam $\left(120^{\circ}\right)$ and IBMSG-modelled fan.

The total pressure distribution is also compared against the passage data from Wartzek's experiment [19] and the URANS simulation. The circumferential distributions are extracted at three axial locations $\left(S_{1}-S_{3}\right.$ shown in Figure 2, representing, respectively, the rotor entry, rotor exit and stator exit. The data at these three locations can provide a detailed view of the influence of the distortion on each individual component. The influence is characterised by the total pressure ratio (for radial locations) and its relative value (for axial locations). They are defined as the ratio of local total pressure to the inflow pressure,

$$
\pi_{t, r e l}=\frac{\pi_{t}}{\bar{\pi}_{t}}, \pi_{t}=\frac{p_{t}}{p_{t, \text { inlet }}} .
$$


This offers a general view of the total pressure variation in the circumferential direction at the rotor entry and exit, as well as the stator exit. In Figure 8, the rotor rotates from the right to the left and the results of the experiment, URANS simulation and RANS (SA model) simulation with IBM are included. The overall trend in all three cases is captured. The relative total pressure ratio increases from frames (a) to (c) within the separation region, meaning that the distortion recovers downstream of the beam, from the rotor inlet to the stator outlet. However, this distortion is maintained through to the stator exit.

In frame (a), it should be noticed that, upstream of the rotor, the relative total pressure ratio from the RANS with IBM is slightly higher than is observed in the other two cases. This discrepancy may be due to the hypothesis of infinite blades, which seems to suppress the separation more than finite blades. To investigate the separation behaviour, an entry side and exit side are defined, highlighting the beam installation region. These two lines also represent the rotor entry and exit boundaries. Around this region, the separation detected by the experiment (approximately $\left.100^{\circ}\right)$ is smaller than the 'entry-exit' labelled beam region $\left(120^{\circ}\right)$ because the low pressure downstream narrows the separation. However, the predictions from both RANS and URANS are much larger at the rotor inlet in frame (a). This problem could be tackled by LES in the future. Liu et al. [31] also found that the Spalart-Allmaras model predicts much larger separation bubbles in compressors. Hence, an innovative modification method was proposed based on helicity in order to consider turbulence energy backscatter, which can be substantial in the region of corner separation in compressors [31,32]. Both of these could be potential methods to improve the accuracy of the simulation of fan-distortion interactions. Interestingly, a W-shape was detected upstream of the rotor in Wartzek's experiment [19] which seems to be caused by the corner vortices and the separation line shear layer at the beam edge [33]. These intensify the total pressure loss at the edges of the separation region, although in the centre, the loss still remains at the original level (Figure 9). However, this is not shown in the present numerical simulation because the RANS method is not able to resolve such unsteady vortices.

Downstream of the rotor in frame (b), although the distribution from RANS with IBM is slightly different in terms of the amplitude, it indeed captures the feature of fan rotation. The 
rotor increases the total pressure ratio at the exit side compared to the entry side. In both

frames (b) and (c), the intermittency of the total pressure at each blade region cannot be captured, compared with the result from URANS. This is because the blade geometry is smeared when using the IBMSG method.

The circumferential distributions at different radial locations are also compared. Three different radial locations are chosen; $10 \%, 50 \%, 90 \%$ of the annulus height at the stator outlet. In Figure 10, although the general trend is captured by the RANS with IBM, it should be noticed that the prediction at the hub $(10 \%)$ is the most accurate. The reason could be that, at the midspan, the infinite blade hypothesis would dominate the impact on the blade performance, and thus intensify the total pressure recovery; at the shroud, however, the SA modelled distortion would have a stronger defect that overpredicts the total pressure loss. In terms of the separation influence downstream, it appears that the biggest variation is at the hub, whereas such a variation is almost invisible at the shroud.

To validate how the IBM beam and IBMSG-modelled fan characterise the separation behaviour, the mass flux distribution is depicted in Figure 11. This includes the results from both the mesh resolved case and the modelled case. In this figure, the radial location is the relative blade height; the mass flux is referenced to the area average. The data were extracted at a distance of $10 \%$ of the blade chord upstream of the rotor entrance. It is obvious that the momentum deficit occurs between $R=0$ and $1.5 H$, where the beam is installed.

These result demonstrate that, although the separation affects the total pressure distributions across the rotor and stator, the IBMSG-modelled rotor can, at least, still accurately capture the main flow features upstream the rotor compared to the experiment and resolved case. This means that separated flows do not have an obvious impact on the application of the IBMSG method.

\section{FAN INFLUENCE ON DISTORTION}

\subsection{Fan location}

The effects of fan location are discussed in this section. To explore how the distance between fan and the distortion generator will affect the distortions, the original blade is moved 
downstream to two different locations: a distance of a half-chord and a full chord, corresponding to $x=5.2 / 6.2 / 7.2 H$.

Figure 12 illustrates the time-averaged axial velocity contour and Q-isosurface of these three cases. A detailed comparison (Figure 13) shows that the original location has the highest effect in terms of increasing of the mass flux and total pressure ratio. A blade location which is further away will have less of an influence in suppressing the separation.

To quantify this blade effect, we define a Recovery Factor to describe how well the blade will cope with the intake distortion and improve the flow condition. Firstly, we introduce a mass-weighted total pressure loss $P_{t, l o s s}$,

$$
P_{t, l o s s}=1-\frac{\int p_{t} d \dot{m}}{p_{t, i n} \dot{m}} .
$$

This reflects the total pressure loss at a designated position, e.g., $10 \%$ of the blade chord upstream the leading edge. Then a Recovery Factor is defined as,

$$
R F=1-\frac{P_{t, l o s s}}{P_{t, l o s s, N F}},
$$

where $N F$ means 'no fan'. This quantifies the percentage of the total pressure recovery when a fan is installed compared to the single duct without a fan. These RFs for the fan at different locations are depicted in Fig. 14.a, and all of the data are extracted from the same axial location $x=4.5 \mathrm{H}, 10 \%$ of the blade chord upstream of the original blade leading edge. Hence, it is obvious that the fan nearer to the intake will considerably improve the total pressure ratio. This is reasonable because a nearer fan can stop the development of the separation much earlier.

\subsection{Inlet Distortion}

The effects of the various degrees of distortion are discussed in this section. To simulate these distortions, two types of bevelled beams were installed at the same location with the 
original case conditions. Figure 15 illustrates the flow distributions of these cases, where both the influence of fan and that of the beam can be compared.

To quantify the effect of these distortions, the relative total pressure loss is illustrated in Figure 16. This figure shows that, when the height of the beam is changed linearly, the distortion-induced loss varies exponentially. This can be also demonstrated by the case of the flow distribution without a fan in Figure 17.

In this figure, the original distortion (or full-scale beam) has a remarkable influence on the main stream, compared to the other two cases. The mass flux is completely redistributed throughout the channel, and the main flow sees a nearly $20 \%$ change in its magnitude. In contrast, the distortion does not have a very great impact on the main flow when using the $1 / 2 H$ and $1 / 4 H$ beams. The total pressure loss is also significant for the full-scale beam, at nearly $11 \%$ within the separation region and $2 \%$ for the main flow. Figure 18 shows how the blade responds to these various distortions.

Evidently, the fan is capable of alleviating the distortions. The effect for the original beam is the most significant, showing a $20 \%$ increase in mass flux and a $2 \%$ increase in total pressure. The effects for the $1 / 2 H$ and $1 / 4 H$ beams are minor. Equation 7 is used to quantify this effect and Figure 14.b shows the Recovery Factor of the fan when coping with these distortions. Obviously, the improvement due to the fan for the full-scale beam is remarkable, at around $44 \%$. This proves that the proposed blade is capable of reducing the intake distortion, even if the flow condition has been significantly changed by the full-scale beam.

\section{CONCLUSION}

In the present research, the distortion generator is modelled using IBM; both the rotor and stator blades are approximated by the IBMSG method. The validation of the modelled components demonstrates that this mixed-fidelity modelling approach can be applied as a suitable tool for predicting the characteristics of intake-fan interaction. The RANS simulation indicates that these models can predict the general trends in performance. At the rotor inlet, exit and stator exit, the total pressure distributions agree well with the experimental data. It should be noted that the radial distribution at the stator exit has some differences due to the 
infinite blade assumption. Despite this, generally, the trend of the distortion development in the axial direction can be captured and the true total pressure ratio can be approximated. Hence, we may conclude that the mixed-fidelity modelling method can reflect the behaviour of intake-fan distortion.

This mixed-fidelity method is then applied to the investigation of the fans influence on the intake distortion. Results indicate that the location of fan has a significant influence on intake distortion. The nearer the downstream fan, the stronger the influence in terms of suppressing separation. The fan at the proposed location can recover $45 \%$ of the loss. This is because the rotational fan can accelerate the upstream flow at the casing, thereby reducing the separation bubble and alleviating the distortion.

Various sizes of distortion are also studied. This is achieved by changing the beam height. It is found that the original beam has such a significant influence that even the main flow can be changed by almost $20 \%$. Nevertheless, the proposed blade is still capable of weakening such distortions. The effects of the blade at different beam heights are also quantified with the conclusion that, when the distortion is larger, the fan has a larger effect. This change is non-linear, and when the beam height is reduced to $H / 4$, the Recovery Factor becomes $R F / 7$. This means that a well-designed blade could show substantial power when dealing with any possible intake distortion. Hence, the fan could form an essential component for intake distortion control.

Further studies will include the investigation of the mechanism of fan behaviour in regard to the inlet distortion. This could be done by analysing the Reynolds stresses and turbulent kinetic energy budgets from LES results. These results are expected to provide detailed information about how turbulence is generated, transformed and dissipated within the recirculation region, and how the fan behaves in this process.

\section{Acknowledgements}

The authors acknowledge the computing time on the UK national high-performance computing service ARCHER provided via the UK Turbulence Consortium in the framework of the EPSRC grant EP/L000261/1. This work is also funded by the studentship from Chinese Schol- 
arship Council. The code for this project is provided by the Rolls-Royce plc. All the funding and technical support from these organizations are gratefully acknowledged. Constructive suggestions from James Tyacke are also much appreciated.

\section{Funding}

The Engineering and Physical Sciences Research Council (EPSRC) grant EP/L000261/1. The Chinese Scholarship Council (CSC) No. 201506020121. 


\section{Nomenclature}

$f$ force

$H$ height of the beam

$K$ viscosity coefficient of parallel force

$k_{1}$ coefficient for viscosity distribution

$n$ normal vector to the blade surface

$t$ time or blade thickness

$p$ pressure

$u$ velocity

$x$ x coordinate

$x_{0}$ body/surface coordinate

$\Delta y_{+}$dimensionless wall distance

$\Delta x_{+} \quad$ dimensionless streamwise distance

$\Delta z_{+} \quad$ dimensionless spanwise distance

$\rho$ density

$\alpha, \beta$ feedback forcing coefficients

$\lambda$ blockage factor

$\pi_{t}$ total pressure ratio

$P_{t, \text { loss }} \quad$ mass-weighted total pressure loss

CFD Computational Fluid Dynamics

RF Recovery factor

RANS Reynolds Averaged Navier-Stokes

URANS Unsteady Reynolds Averaged Navier-Stokes

LES Large Eddy Simulation

IBM Immerse Boundary Method

IBMSG Immersed Boundary Method with Smeared Geometry

SA Spalart-Allmaras turbulence model

$k-\omega \quad k-\omega$ turbulence model 


\section{References}

[1] Xie, Z., Liu, Y., Liu, X., Sun, D., Lu, L., and Sun, X., 2017. "Computational model for stall inception and nonlinear evolution in axial flow compressors". Journal of Propulsion and Power, pp. 1-10.

[2] Tucker, P., and Liu, Y., 2006. "Turbulence modeling for flows around convex features". In 44th AIAA Aerospace Sciences Meeting and Exhibit, p. 716.

[3] Liu, Y., Yu, X., and Liu, B., 2008. "Turbulence models assessment for large-scale tip vortices in an axial compressor rotor". Journal of Propulsion and Power, 24(1), pp. 15-25.

[4] Scillitoe, A. D., Tucker, P. G., and Adami, P., 2015. "Evaluation of rans and zdes methods for the prediction of three-dimensional separation in axial flow compressors". In ASME Turbo Expo 2015: Turbine Technical Conference and Exposition, Montreal, Canada, American Society of Mechanical Engineers, pp. V02BT39A041-V02BT39A041.

[5] Liu, Y., Yan, H., Liu, Y., Lu, L., and Li, Q., 2016. "Numerical study of corner separation in a linear compressor cascade using various turbulence models". Chinese Journal of Aeronautics, 29(3), pp. 639-652.

[6] Liu, Y., Yan, H., Lu, L., and Li, Q., 2017. "Investigation of vortical structures and turbulence characteristics in corner separation in a linear compressor cascade using ddes". Journal of Fluids Engineering, 139(2), p. 021107.

[7] Scillitoe, A. D., Tucker, P. G., and Adami, P., 2017. "Numerical investigation of threedimensional separation in an axial flow compressor: The influence of freestream turbulence intensity and endwall boundary layer state". ASME J. Turbomach., 139(2), p. 021011.

[8] Yan, H., Liu, Y., Li, Q., and Lu, L., 2018. "Turbulence characteristics in corner separation in a highly loaded linear compressor cascade". Aerospace Science and Technology, 75, pp. 139-154.

[9] Gourdain, N., Gicquel, L. Y., and Collado, E., 2012. "Comparison of rans and les for prediction of wall heat transfer in a highly loaded turbine guide vane". Journal of propulsion and power, 28(2), pp. 423-433.

[10] Slotnick, J., Khodadoust, A., Alonso, J., Darmofal, D., Gropp, W., Lurie, E., and Mavriplis, 
D., 2014. "CFD vision 2030 study: a path to revolutionary computational aerosciences".

[11] Marble, F. E., 1964. "Three-dimensional flow in turbomachines". High Speed Aerodynamics and Jet Propulsion, 10, pp. 83-166.

[12] Xu, L., 2002. "Assessing viscous body forces for unsteady calculations". In ASME Turbo Expo 2002: Power for Land, Sea, and Air, American Society of Mechanical Engineers, pp. 323-331.

[13] Peskin, C. S., 2002. "The immersed boundary method". Acta numerica, 11, pp. 479-517.

[14] Fadlun, E., Verzicco, R., Orlandi, P., and Mohd-Yusof, J., 2000. "Combined immersedboundary finite-difference methods for three-dimensional complex flow simulations". Journal of computational physics, 161(1), pp. 35-60.

[15] Defoe, J. J., and Spakovszky, Z. S., 2013. "Effects of boundary-layer ingestion on the aero-acoustics of transonic fan rotors". ASME J. Turbomach., 135(5), p. 051013.

[16] Lieser, J., Biela, C., Pixberg, C., Schiffer, H.-P., Schulze, S., Lesser, A., Kähler, C., and R, N., 2011. "Compressor rig test with distorted inflow using distortion generators".

[17] Niehuis, R., Lesser, A., Probst, A., Radespiel, R., Schulze, S., Kähler, C., Spiering, F., and Kroll, N., 2013. "Simulation of nacelle stall and engine response".

[18] Übelacker, S., Hain, R., and Kähler, C. J., 2016. "Flow investigations in a stalling nacelle inlet under disturbed inflow". In Advances in Simulation of Wing and Nacelle Stall. Springer, pp. 271-283.

[19] Wartzek, F., Holzinger, F., Brandstetter, C., and Schiffer, H.-P., 2016. "Realistic inlet distortion patterns interacting with a transonic compressor stage". In Advances in Simulation of Wing and Nacelle Stall. Springer, pp. 285-302.

[20] Fidalgo, V. J., Hall, C., and Colin, Y., 2012. "A study of fan-distortion interaction within the nasa rotor 67 transonic stage". ASME J. Turbomach., 134(5), p. 051011.

[21] Barthmes, S., Haug, J. P., Lesser, A., and Niehuis, R., 2016. "Unsteady cfd simulation of transonic axial compressor stages with distorted inflow". In Advances in Simulation of Wing and Nacelle Stall. Springer, pp. 303-321.

[22] Ma, Y., Cui, J., Vadlamani, N. R., and Tucker, P., 2018. "Effect of fan on inlet distortion: Mixed-fidelity approach". AIAA Journal, 56(6), pp. 2350-2360. 
[23] Sirovich, L., 1967. "Initial and boundary value problems in dissipative gas dynamics". The Physics of Fluids, 10(1), pp. 24-34.

[24] Sirovich, L., 1968. "Steady gasdynamic flows". The Physics of Fluids, 11(7), pp. 14241439.

[25] Salathé, E. P., and Sirovich, L., 1967. "Boundary-value problems in compressible magnetohydrodynamics". The Physics of Fluids, 10(7), pp. 1477-1491.

[26] Goldstein, D., Handler, R., and Sirovich, L., 1993. "Modeling a no-slip flow boundary with an external force field". Journal of Computational Physics, 105(2), pp. 354-366.

[27] Cao, T., Vadlamani, N. R., Tucker, P. G., Smith, A. R., Slaby, M., and Sheaf, C. T., 2017. "Fan-intake interaction under high incidence". Journal of Engineering for Gas Turbines and Power, 139(4), p. 041204.

[28] Cao, T., Hield, P., and Tucker, P. G., 2017. "Hierarchical immersed boundary method with smeared geometry". Journal of Propulsion and Power, pp. 1-13.

[29] Watson, R., Cui, J., Ma, Y., and Hield, P., 2017. "Improved hierarchical modelling for aerodynamically coupled systems". In ASME Turbo Expo 2017: Turbine Technical Conference and Exposition, Charlotte, USA, American Society of Mechanical Engineers.

[30] Bitter, M., Wartzek, F., Übelacker, S., Schiffer, H.-P. K., and J, C., 2015. "Characterization of a distorted transonic compressor flow using dual-luminophore pressure-sensitive paint". In 10th Pacific Symposium on Flow Visualization and Image Processing (PSFVIP10), fedOA (Federico II Open Archive), Naples, Italy.

[31] Liu, Y., Lu, L., Fang, L., and Gao, F., 2011. "Modification of spalart-allmaras model with consideration of turbulence energy backscatter using velocity helicity". Physics Letters A, 375(24), pp. 2377-2381.

[32] Tang, Y., Liu, Y., and Lu, L., 2018. "Solidity effect on corner separation and its control in a high-speed low aspect ratio compressor cascade". International Journal of Mechanical Sciences, 142, pp. 304-321.

[33] Wartzek, F., Brandstetter, C., Holzinger, F., and Schiffer, H., 2015. "Response of a transonic compressor to a massive inlet distortion". In European Turbomachinery Conference, Madrid, Spain, Mar, pp. 23-27. 


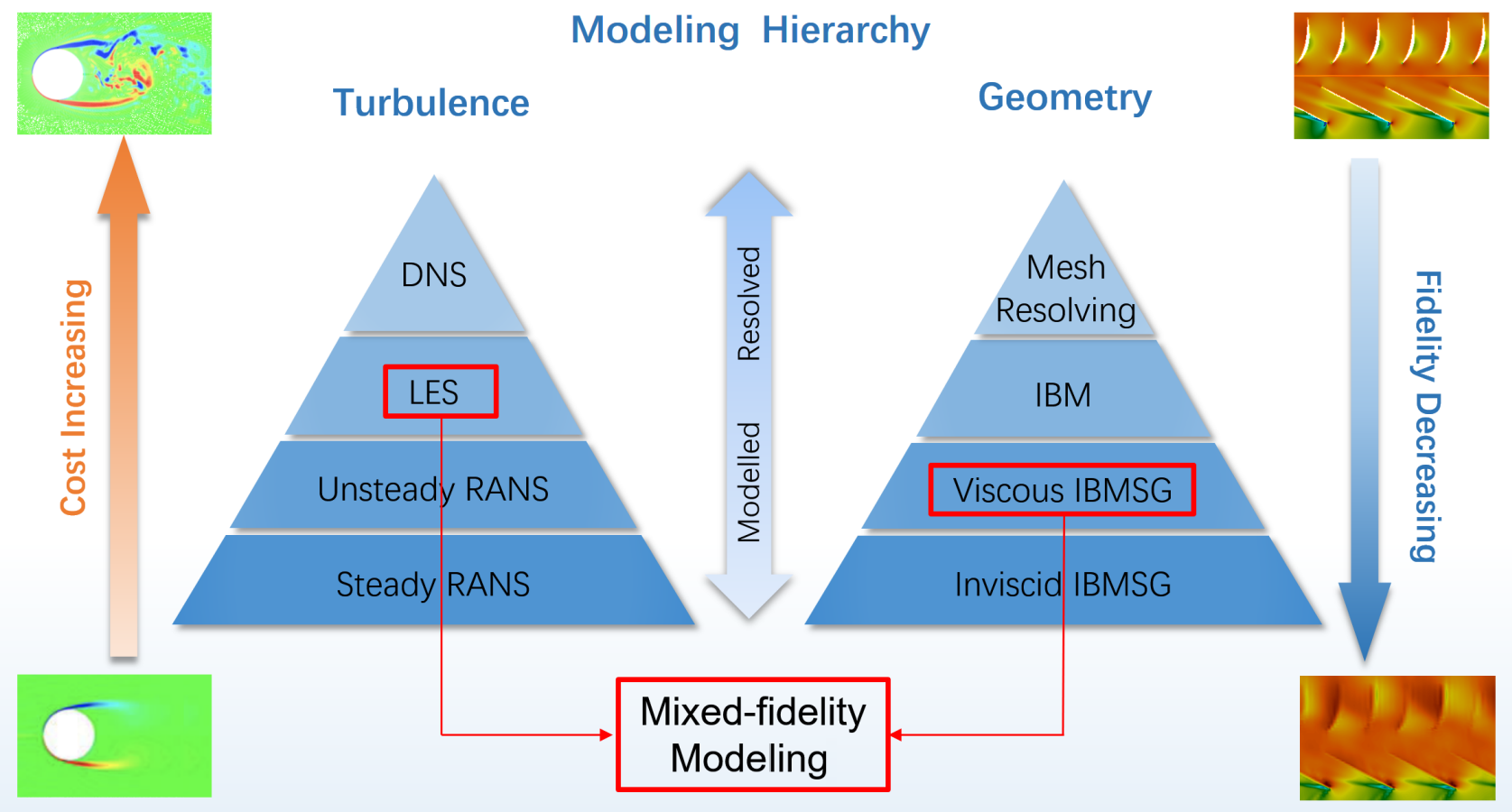

Fig. 1: Hierarchy of turbulence and geometry modelling

\section{Figures}

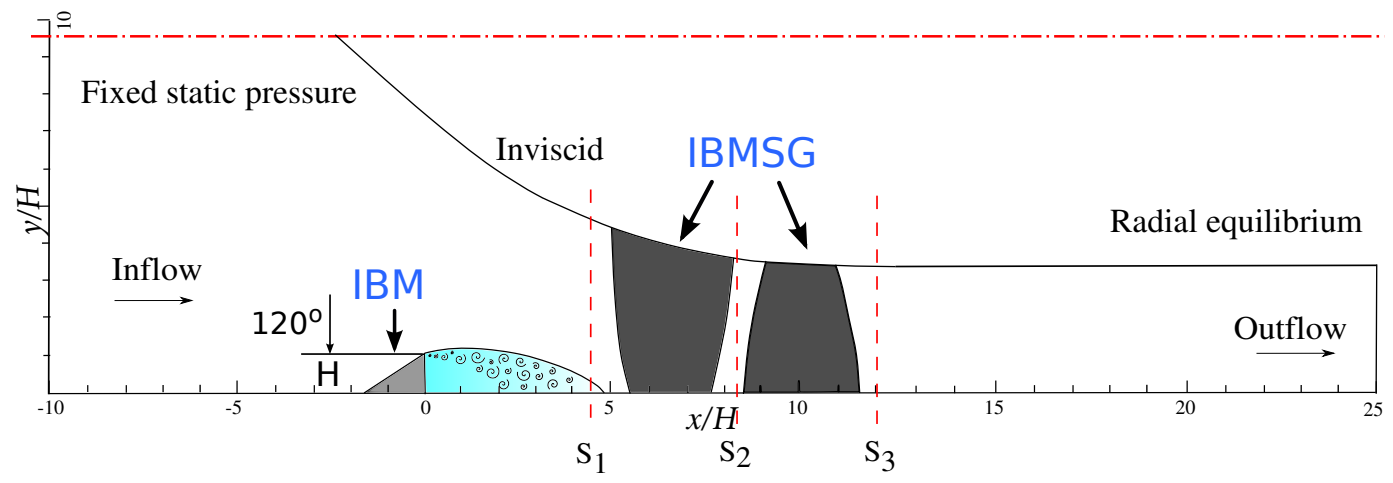

Fig. 2: Sketch of the Darmstadt Rotor test case setup for validation 


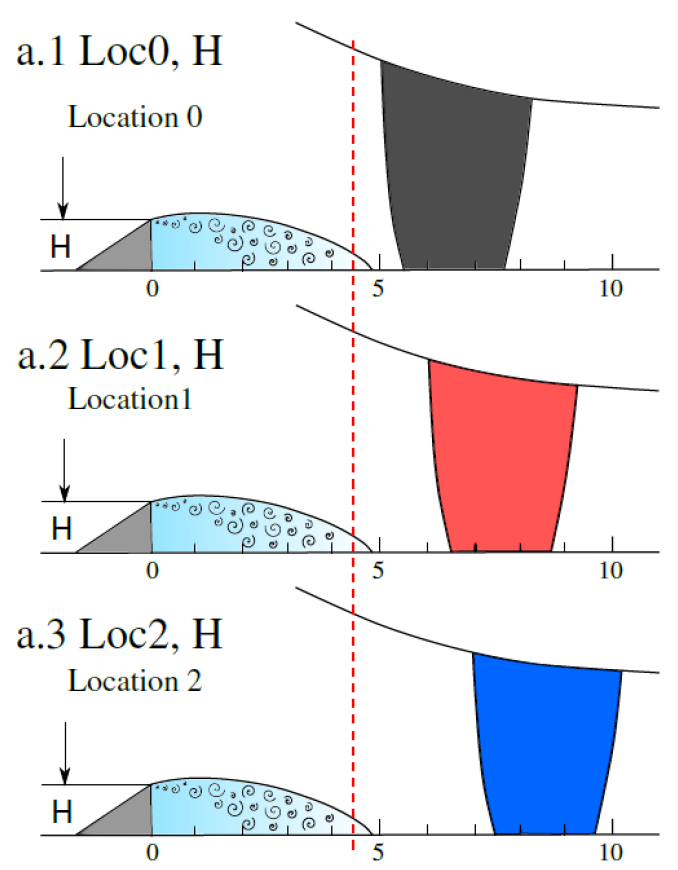

(a) Different Locations

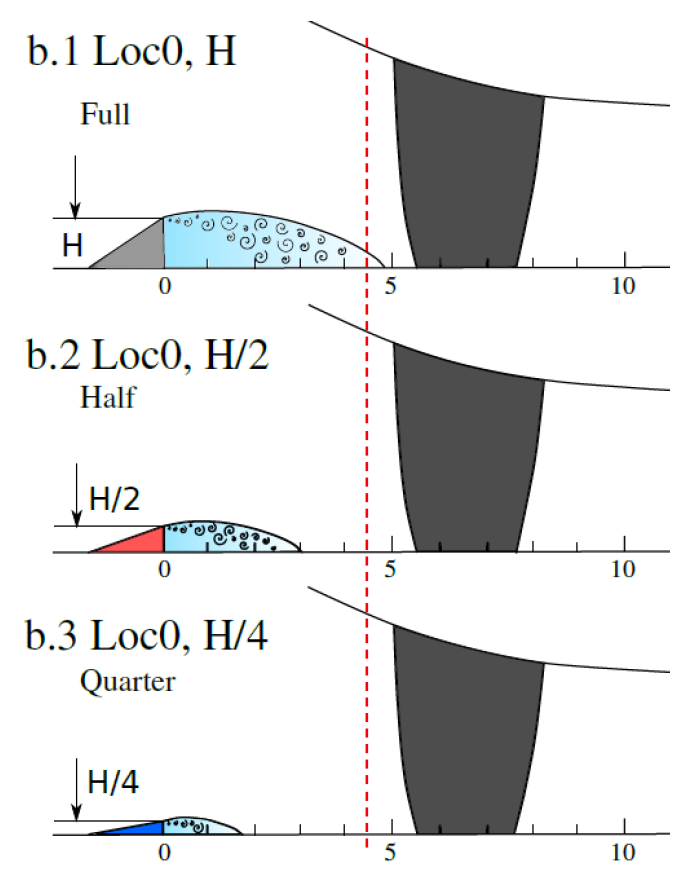

(b) Different beam heights

Fig. 3: Sketch of the test cases for varying (a) fan-locations and (b) beam heights

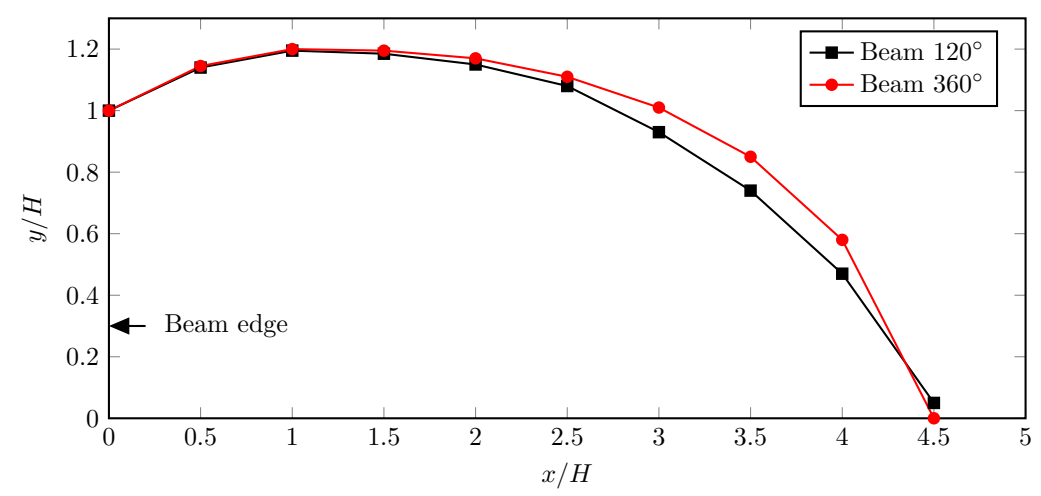

Fig. 4: Inflectional points of the separation bubble

\section{Tables}

Table 1: Key parameters of the Darmstadt Transonic Compressor, from Wartzek [19]

\begin{tabular}{ll}
\hline max. power & $800 \mathrm{~kW}$ \\
max. torque & $350 \mathrm{Nm}$ \\
outer diameter & $0.38 \mathrm{~m}$ \\
hub-to-tip-ratio & 0.51 \\
rotor blades & 16 \\
stator blades & 29 \\
$65 \%$ speed & $13,000 \mathrm{rpm}$ \\
design mass flow & $10.6 \mathrm{~kg} / \mathrm{s}$ \\
\hline
\end{tabular}




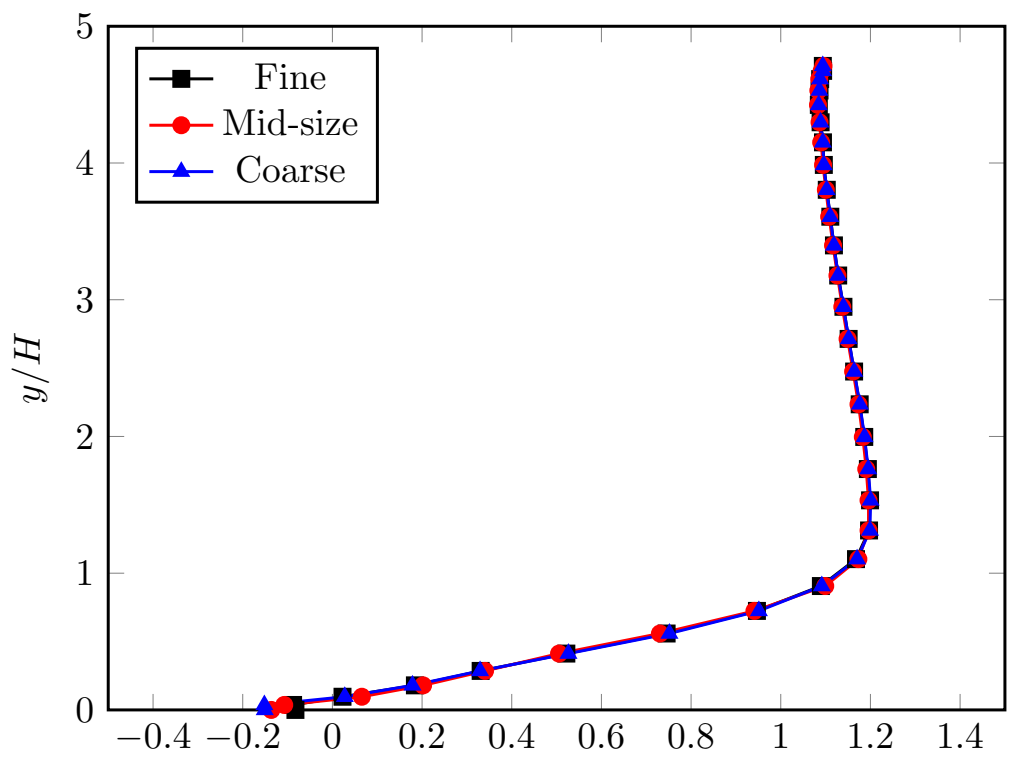

(a) Mass flux

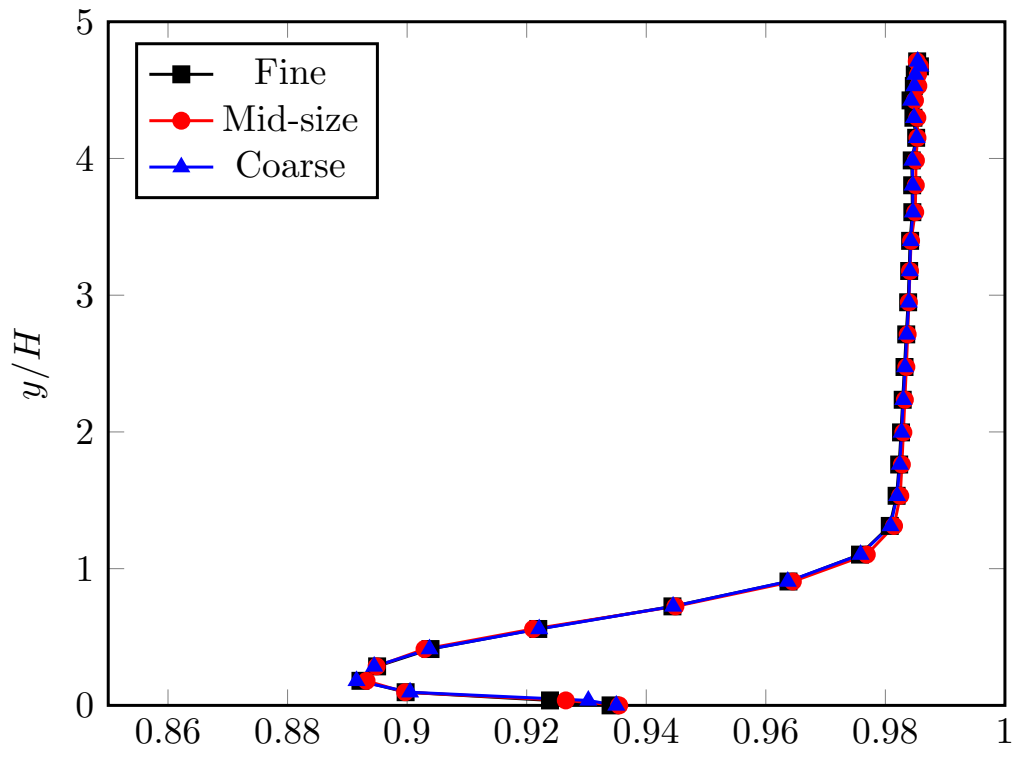

(b) Total pressure ratio

Fig. 5: Prediction of wall-normal profiles of (a) mass flux and (b) total pressure at $x=4.5 \mathrm{H}$ using different meshes

Table 2: Grid spacing for the region between the beam and fan

\begin{tabular}{lllll}
\hline Grid size & $\Delta x^{+}$ & $\Delta y^{+}$ & $\Delta z^{+}$ & Number of nodes \\
Coarse grid & 150 & 1 & 100 & $8,600,100$ \\
Fine grid & 75 & 1 & 30 & $59,371,200$ \\
\hline
\end{tabular}




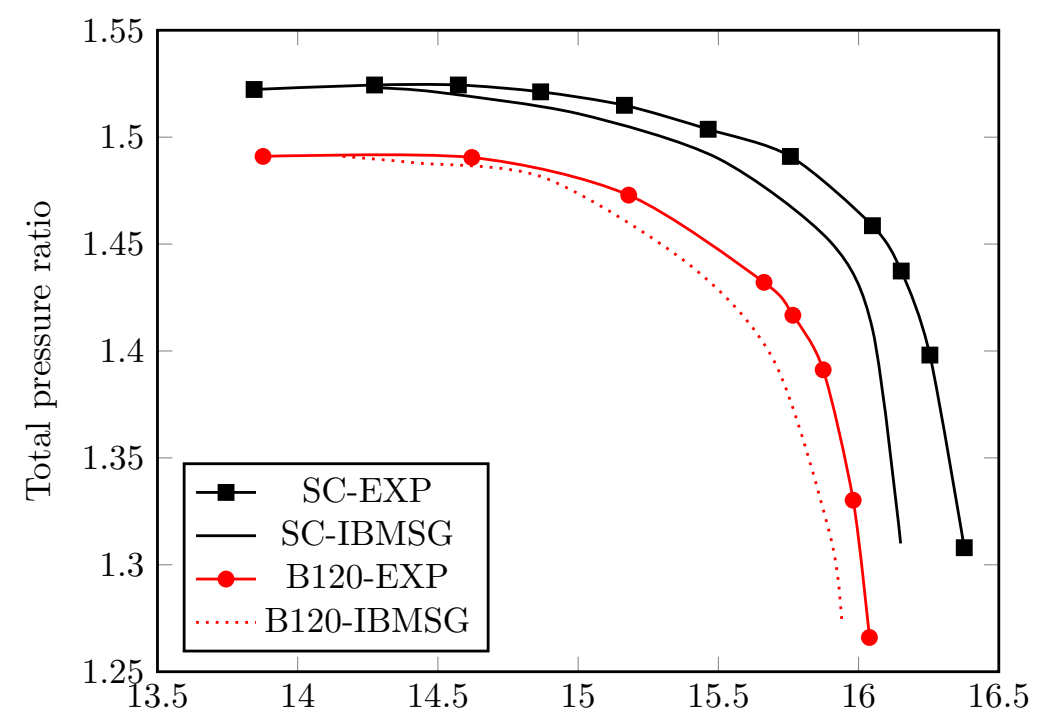

(a) Massflow rate $(\mathrm{kg} / \mathrm{s}, 100 \% \mathrm{RS})$

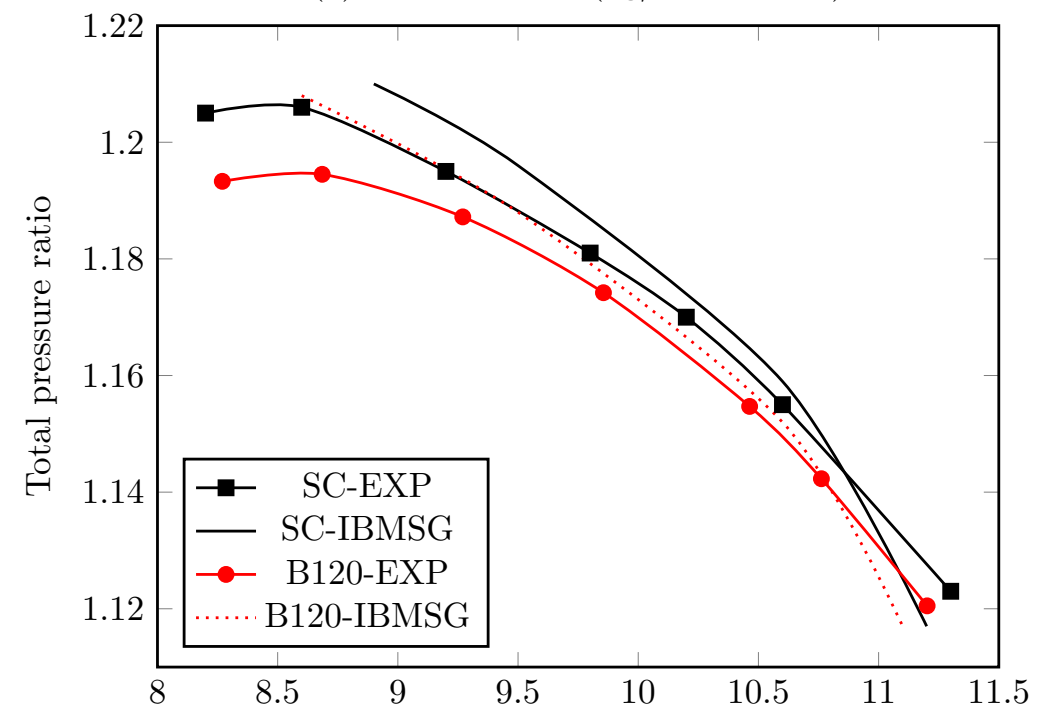

(b) Massflow rate $(\mathrm{kg} / \mathrm{s}, 65 \% \mathrm{RS})$

Fig. 6: Performance map of the Darmstadt Rotor at (a) $100 \%$ speed and (b) $65 \%$ speed. SC represents Smooth Casing and B120 represents $120^{\circ}$ beam. 


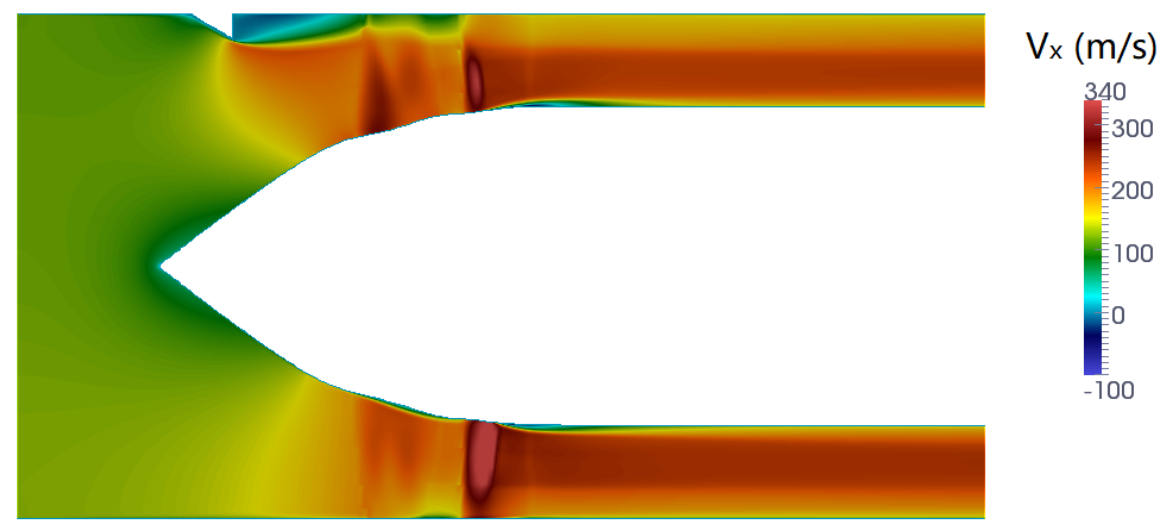

(a) Axial velocity distribution on the meridional plane

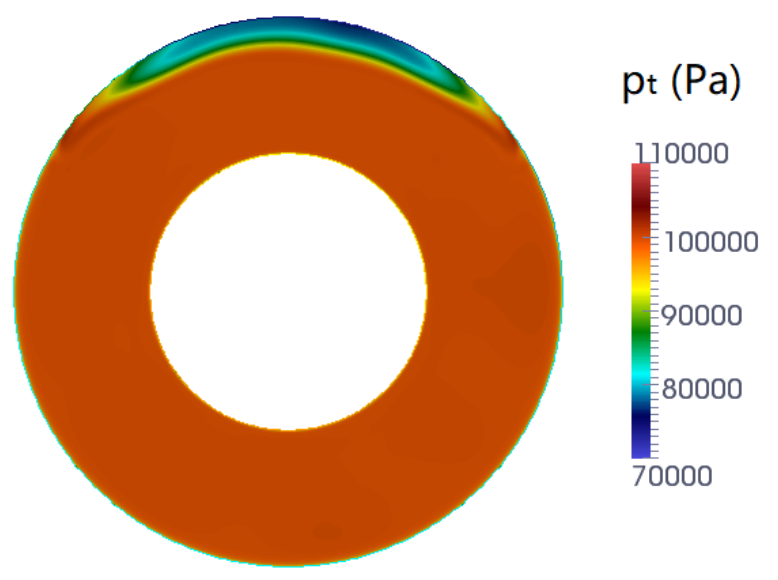

(b) Total pressure distribution on the cross section at upstream the rotor trailing edge

Fig. 7: Contours of (a) axial velocity on meridonial plane and (b) Total pressure upstream of rotor trailing edge at $100 \%$ speed 


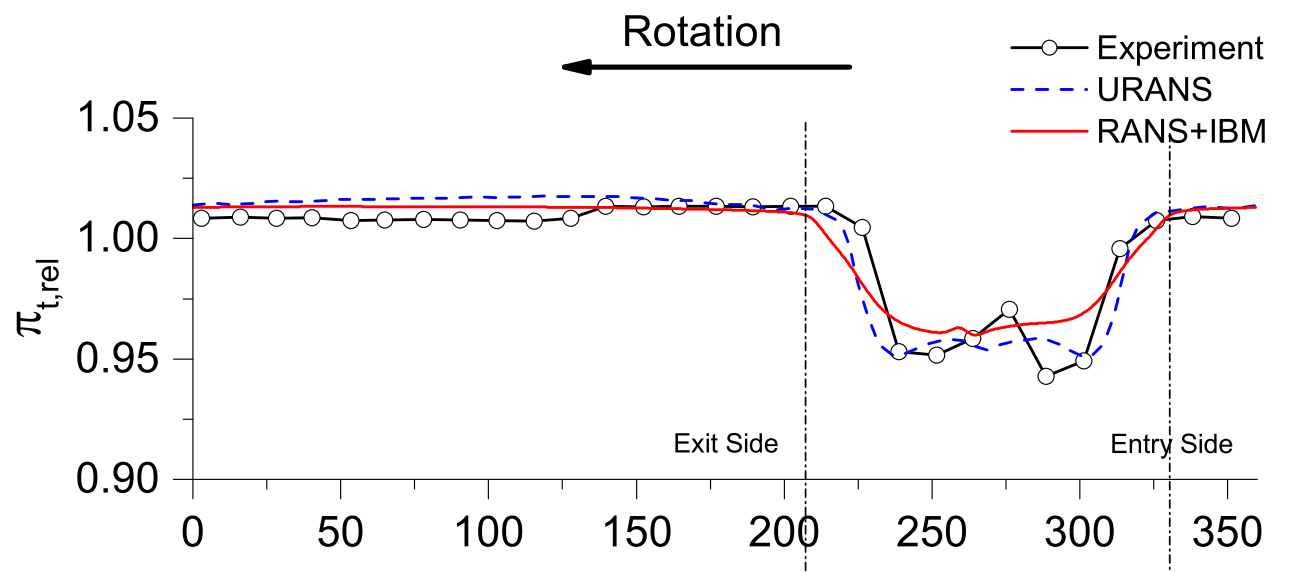

(a) Rotor Inlet

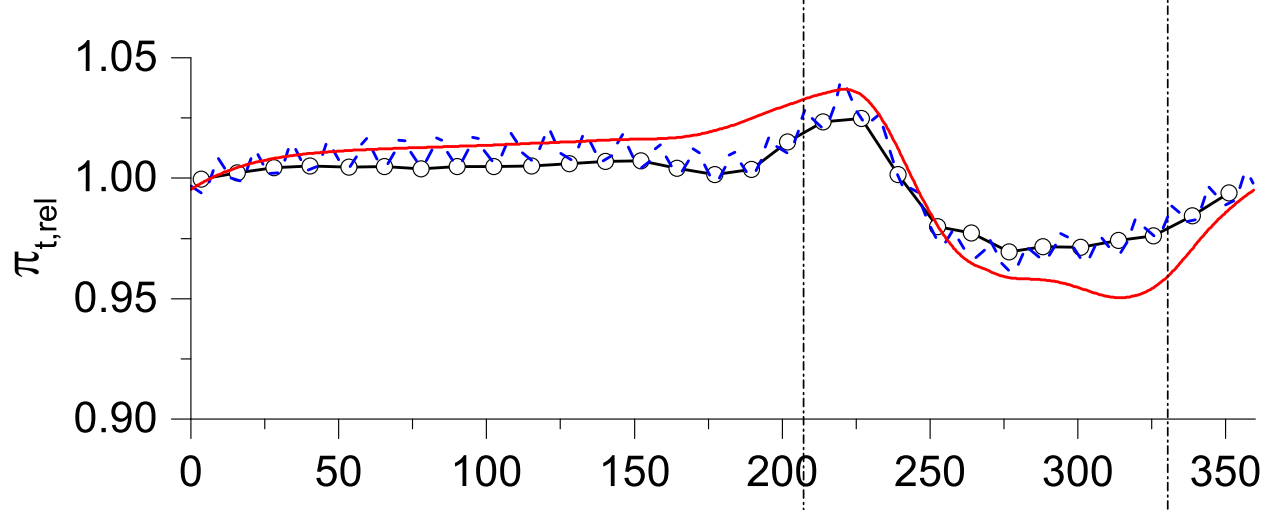

(b) Rotor Outlet

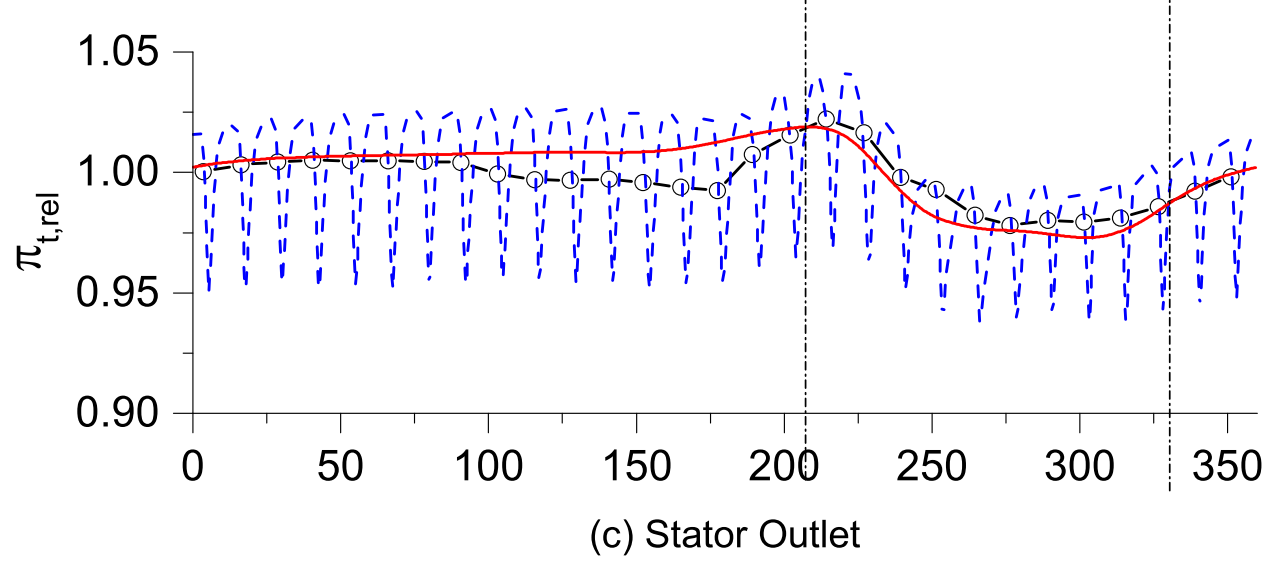

Fig. 8: Circumferential variation of the relative total pressure ratio at three axial stations: (a) rotor inlet; (b) rotor outlet; (c) stator outlet at $100 \%$ speed, radially averaged by area 


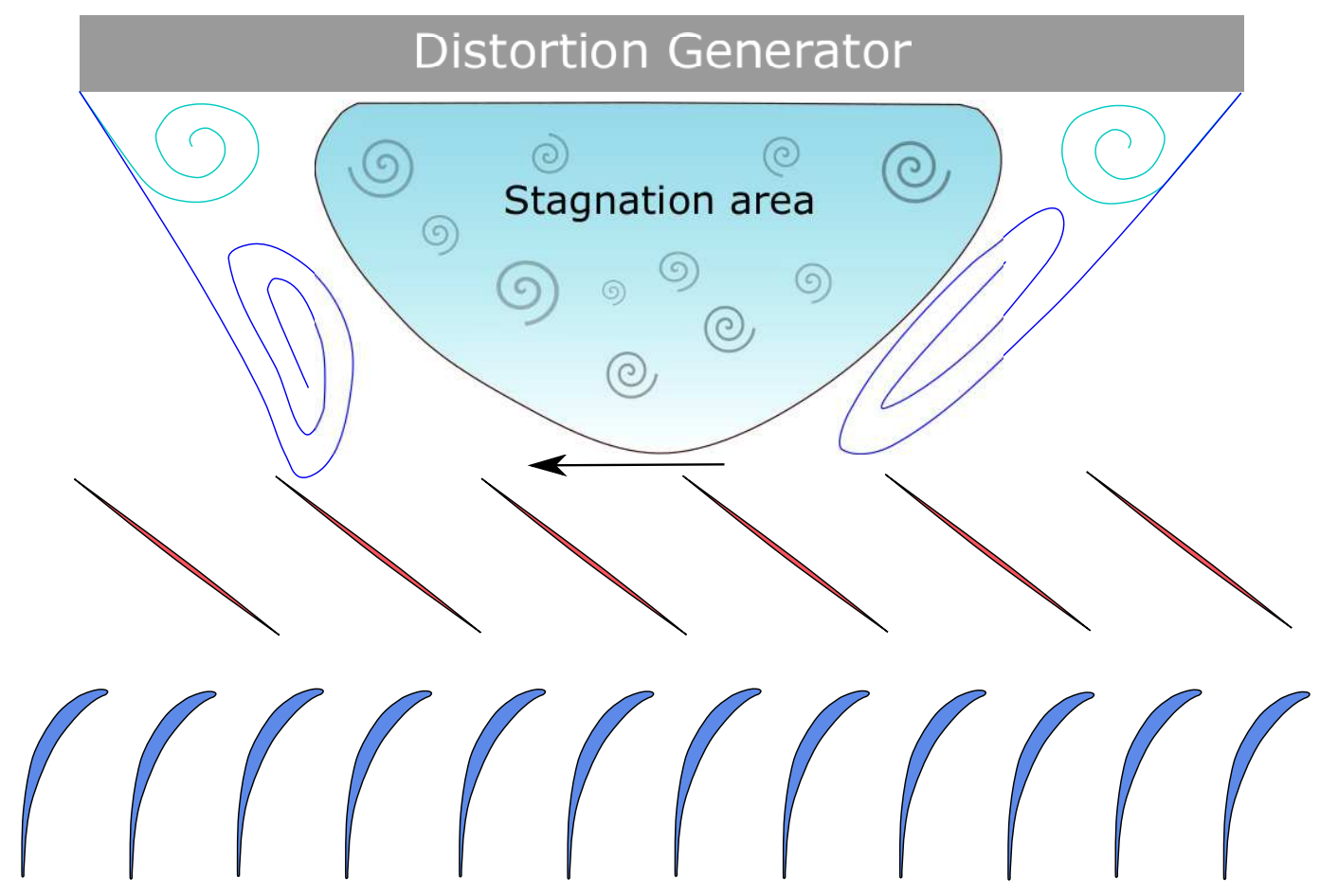

Fig. 9: Separation region downstream the beam 


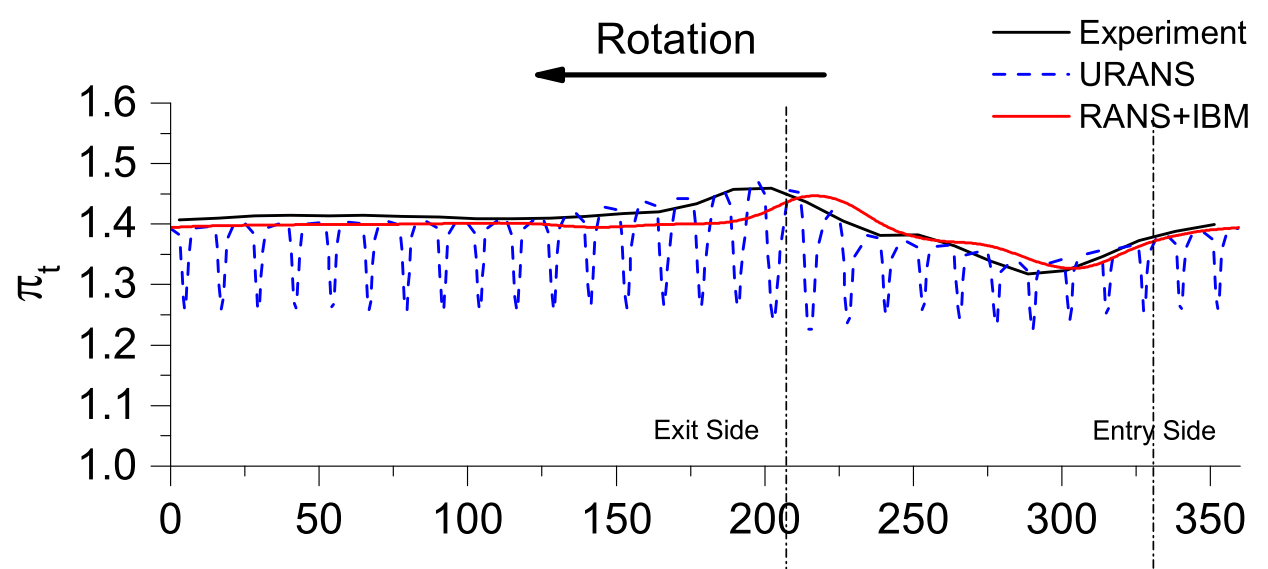

(a) Hub

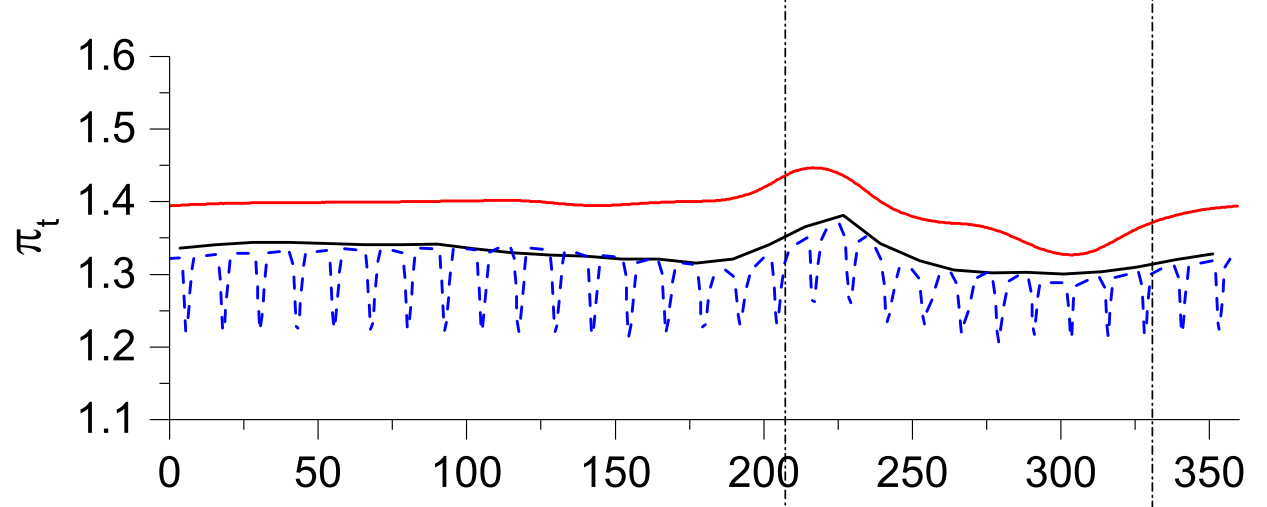

(b) Midspan

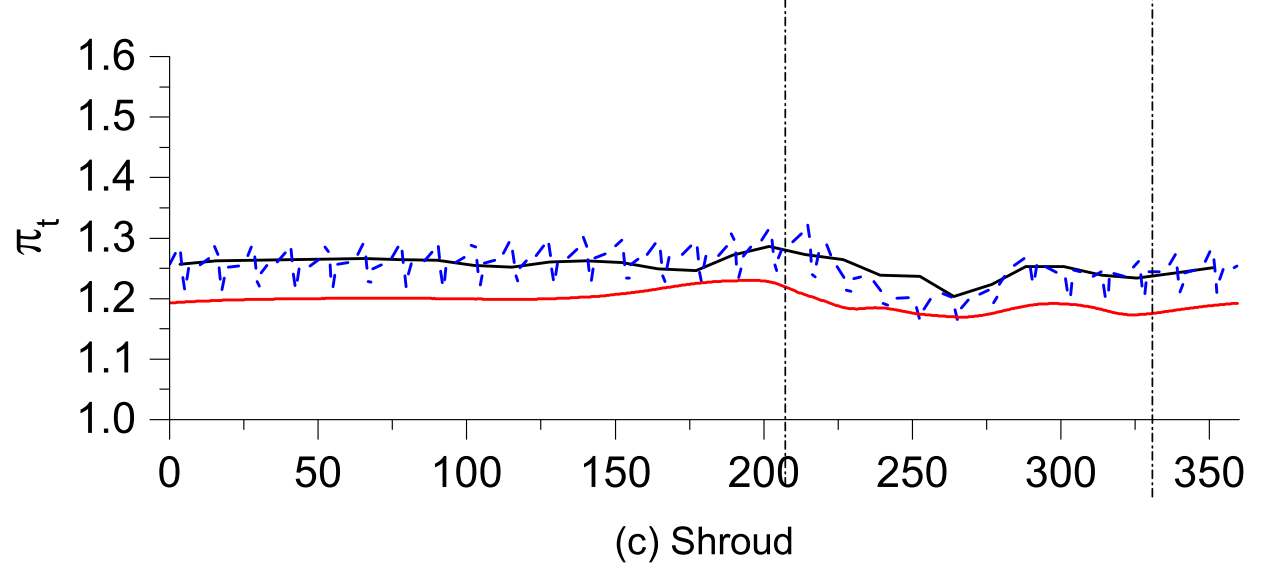

Fig. 10: Circumferential variation of the total pressure ratio at the stage exit at $100 \%$ speed. Three radial locations are shown: (a) 10\%, (b) 50\%, and (c) $90 \%$ of the annulus height. 


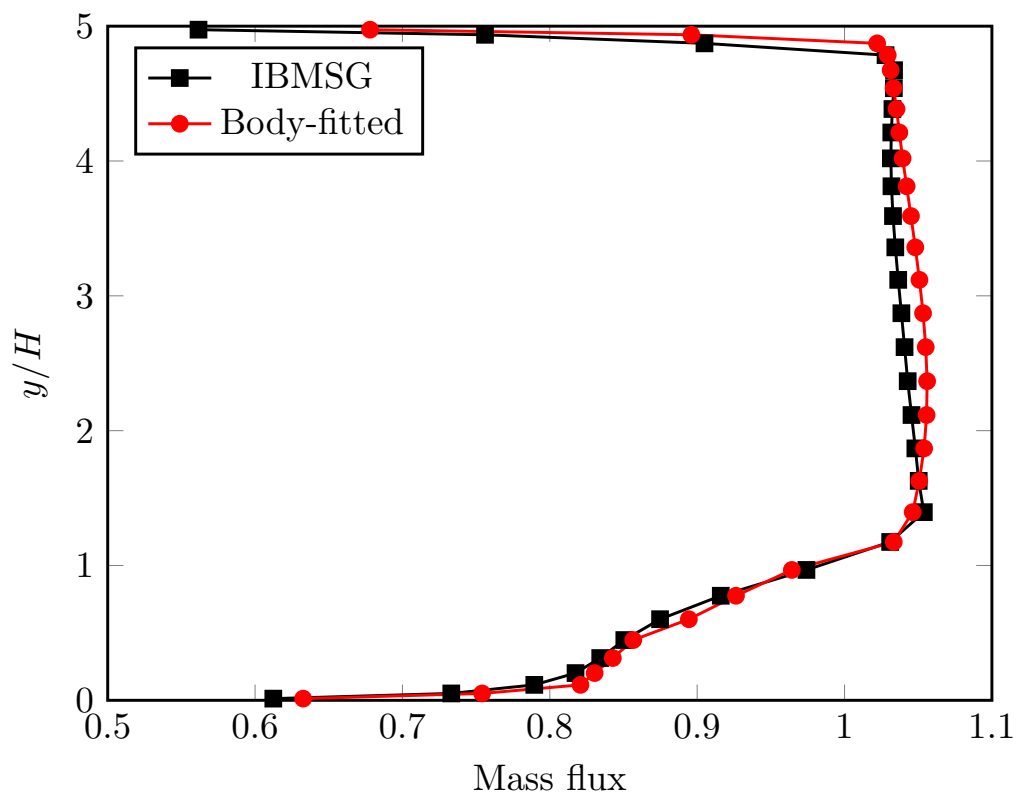

Fig. 11: Radial distribution of mass flux from IBMSG modelling and Resolved cases 


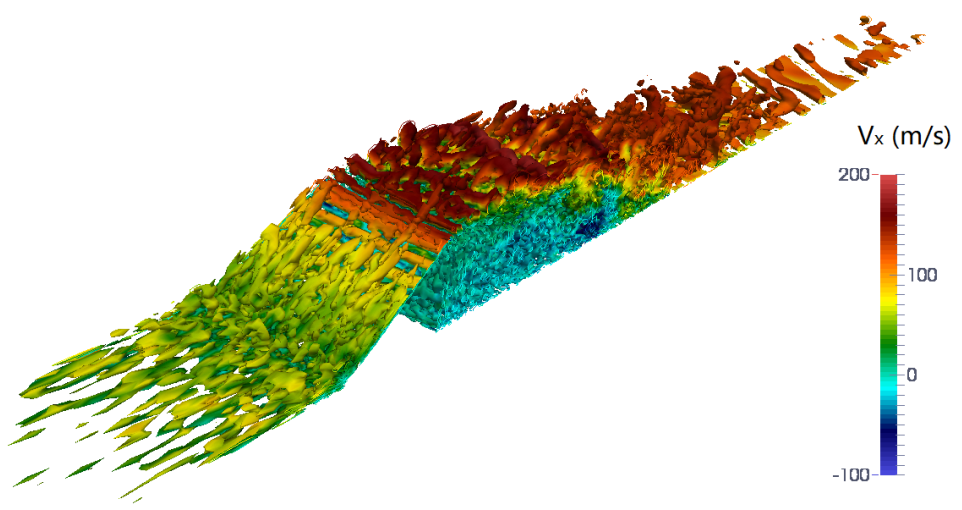

(a) Location $1 x=6.2 H$

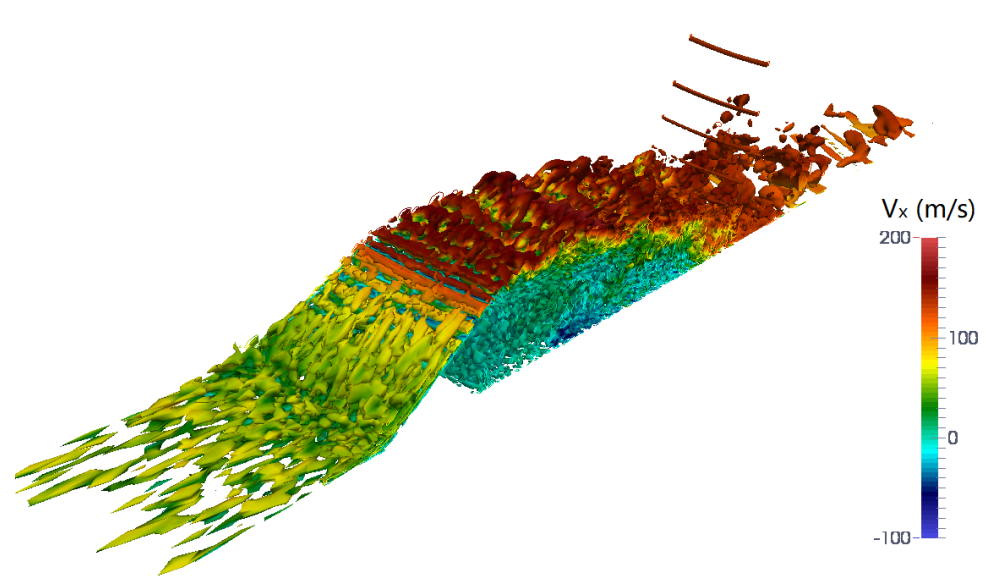

(b) Location $1 x=7.2 \mathrm{H}$

Fig. 12: Q-isosurfaces $\left(Q=1 \times 10^{7}\right)$ coloured with axial velocity for different fan-locations (a) $x=6.2 \mathrm{H}$ and $(\mathrm{b}) x=7.2 \mathrm{H}$ 


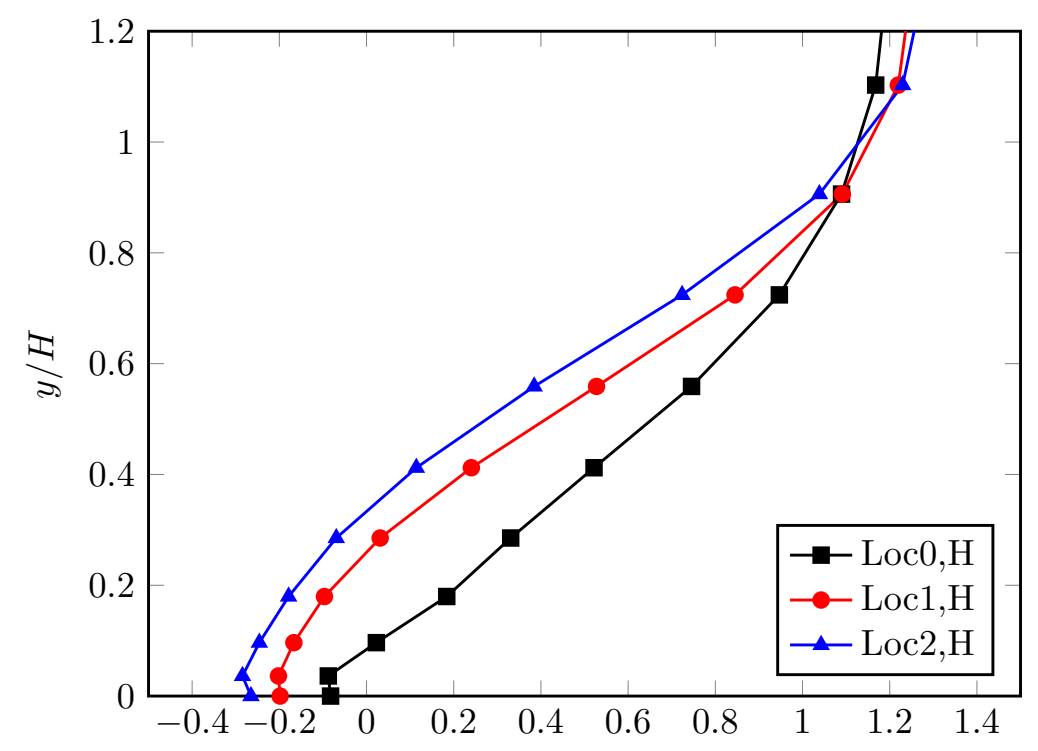

(a) Mass flux

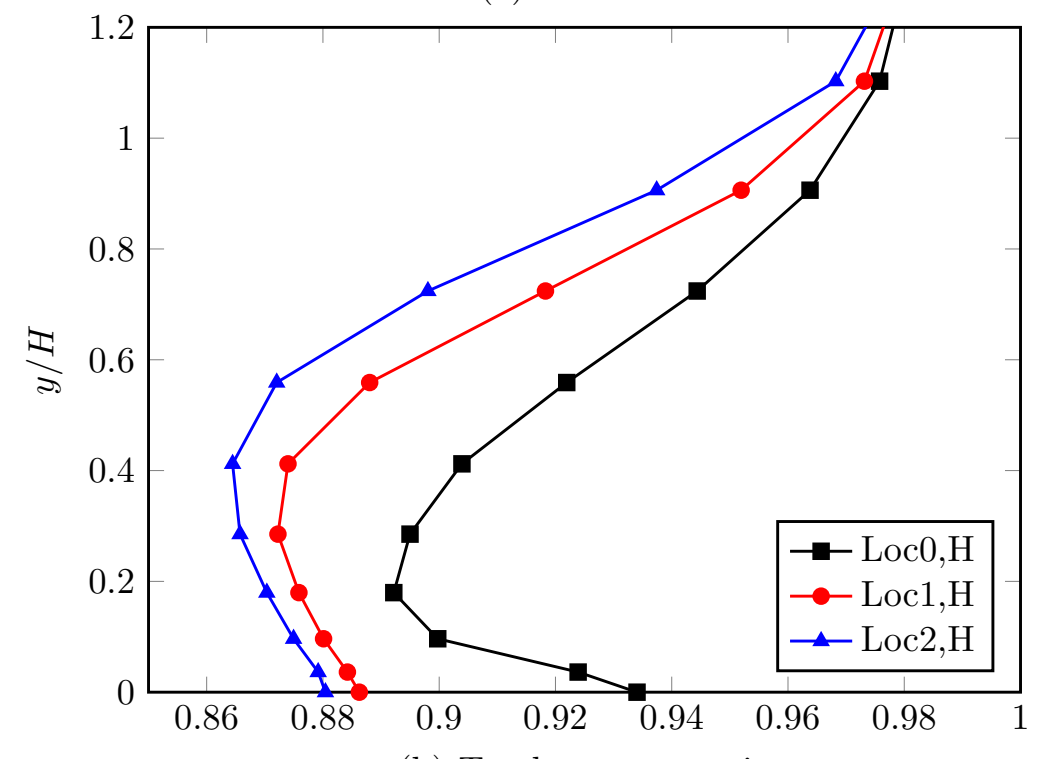

(b) Total pressure ratio

Fig. 13: Effects of different blade locations on (a) mass flux and (b) total pressure ratio 


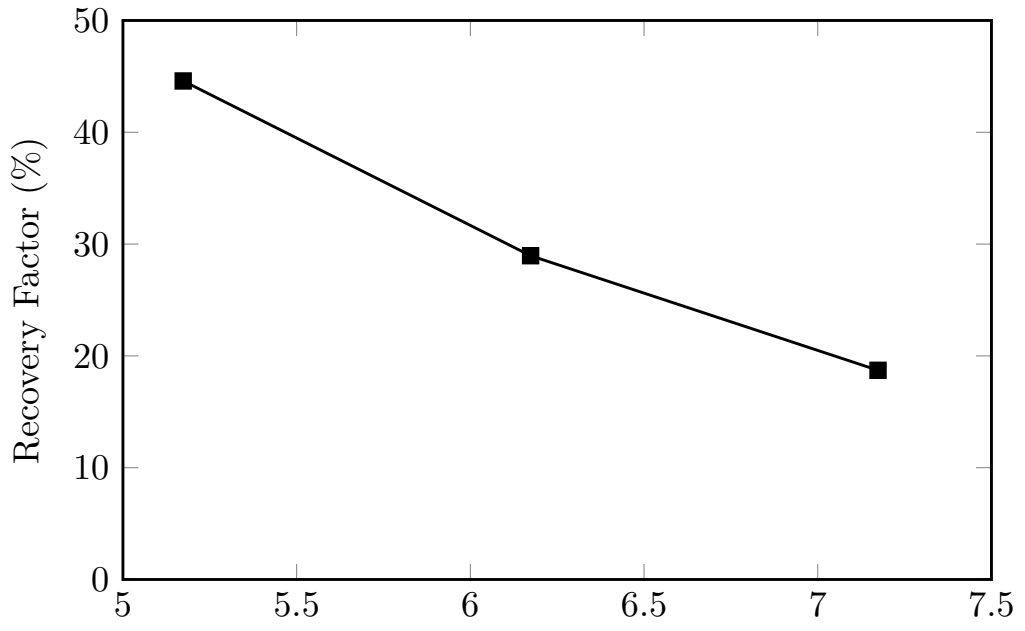

(a) Fan location $x / H$

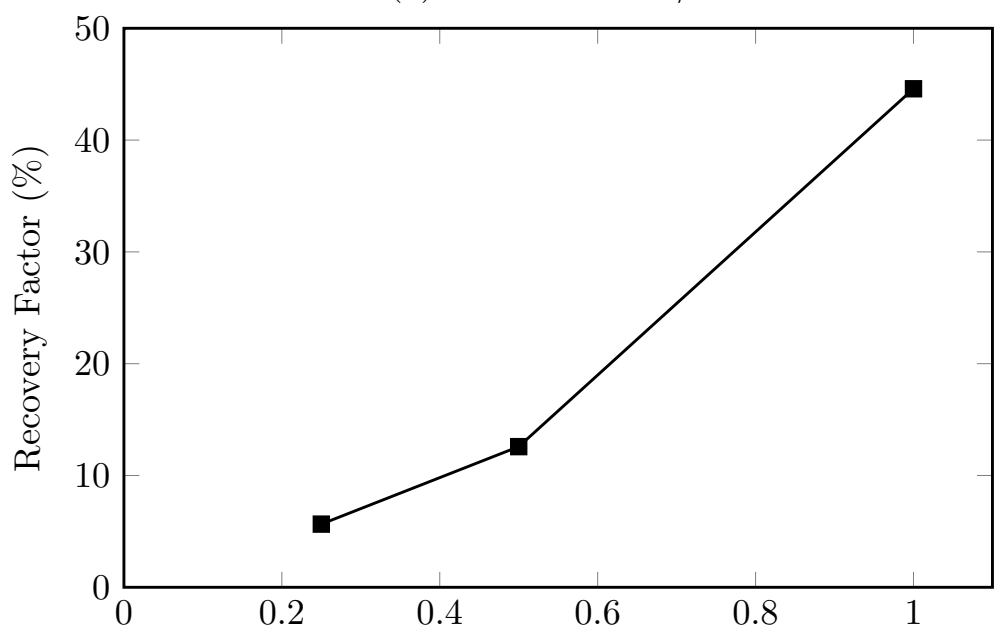

(b) Beam height $y / H$

Fig. 14: Recovery Factor with varying (a) fan-locations and (b) beam heights 


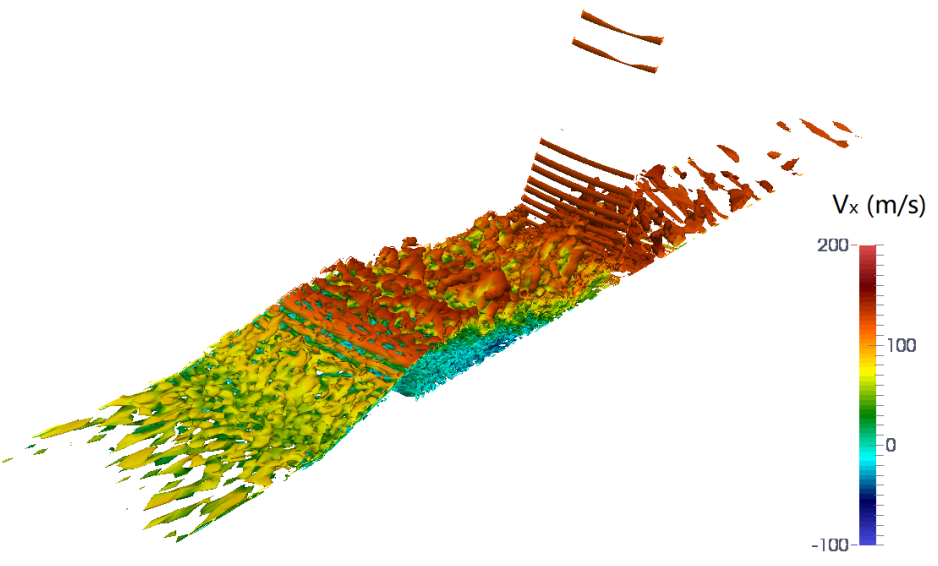

(a) $1 / 2 \mathrm{H}$, with fan

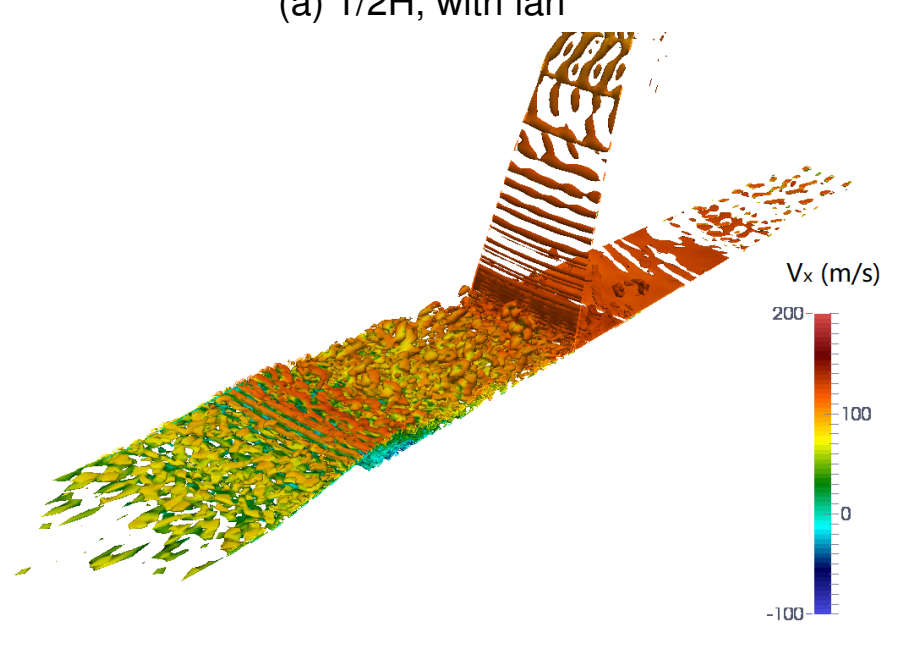

(b) $1 / 4 \mathrm{H}$, with fan

Fig. 15: $Q$-isosurfaces $\left(Q=1 \times 10^{7}\right)$ coloured with axial velocity for different beam heights

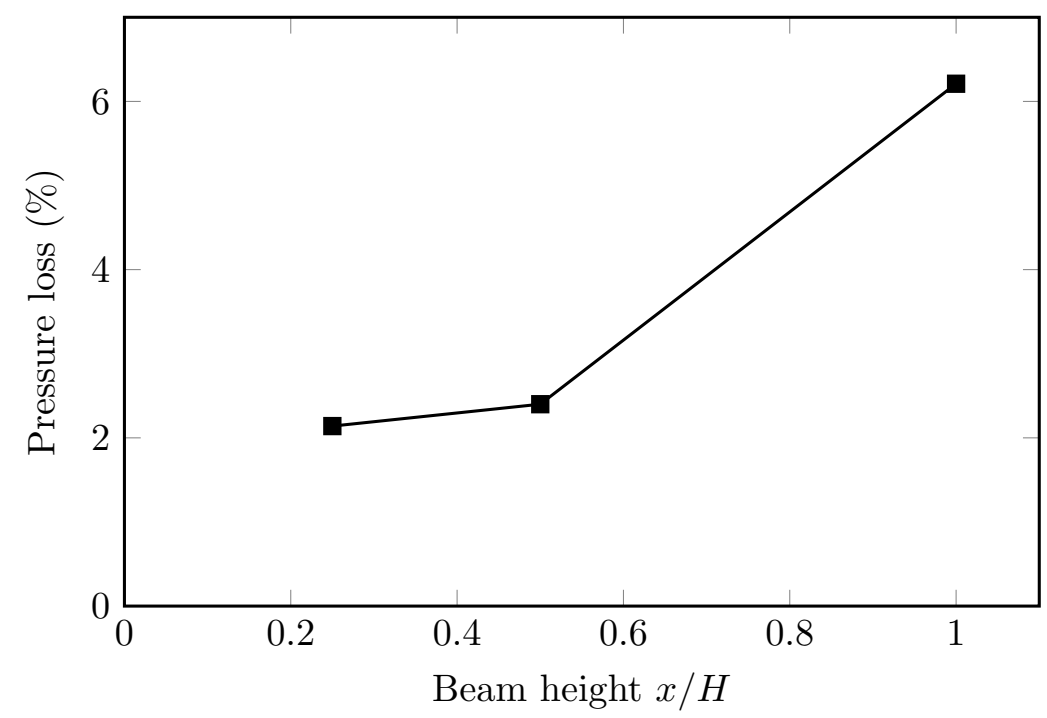

Fig. 16: Total pressure loss with increasing beam height 


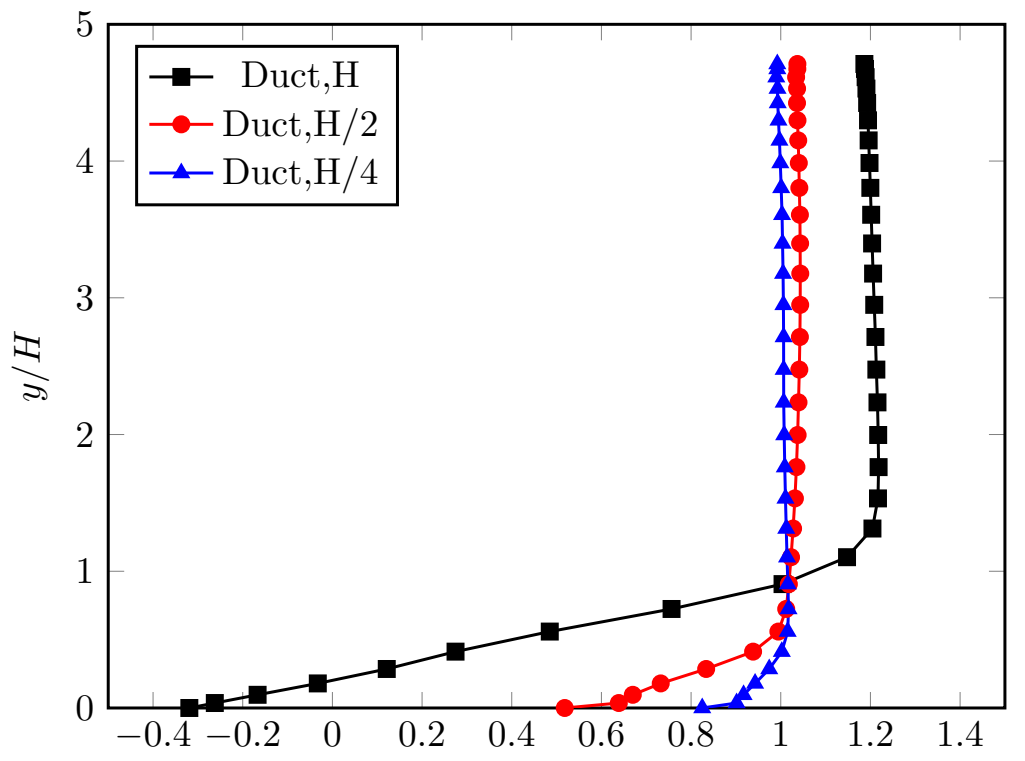

(a) Mass flux

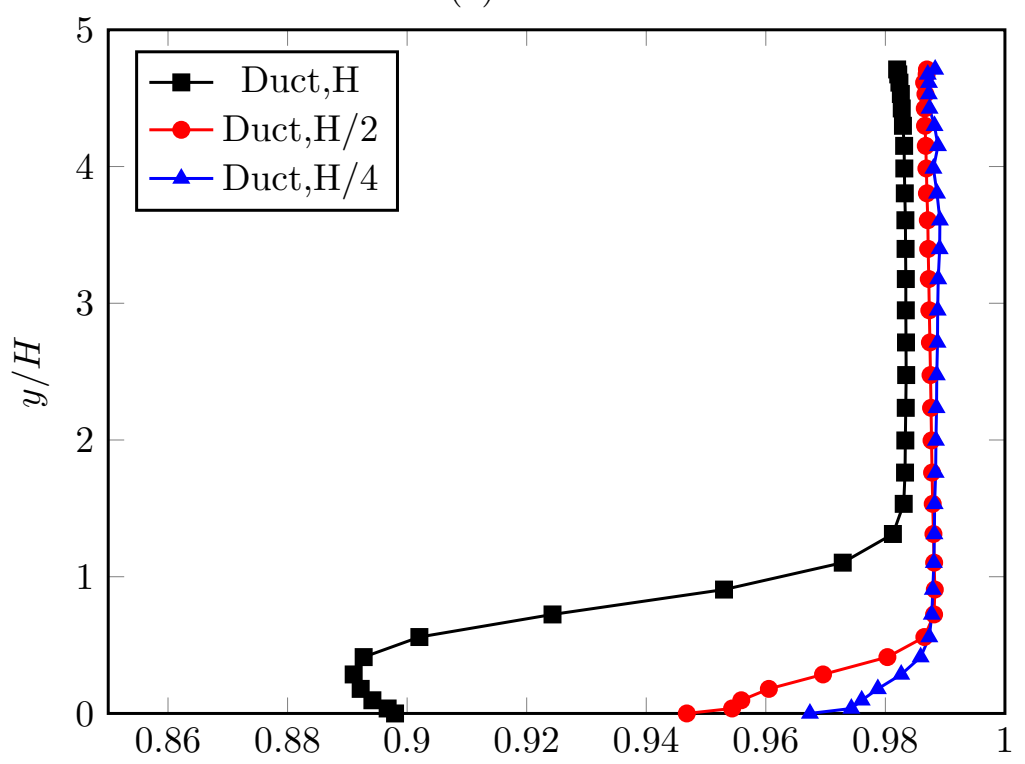

(b) Total pressure ratio

Fig. 17: Effect of different degrees of distortion on (a) mass flux and (b) total pressure ratio in the absence of fan 


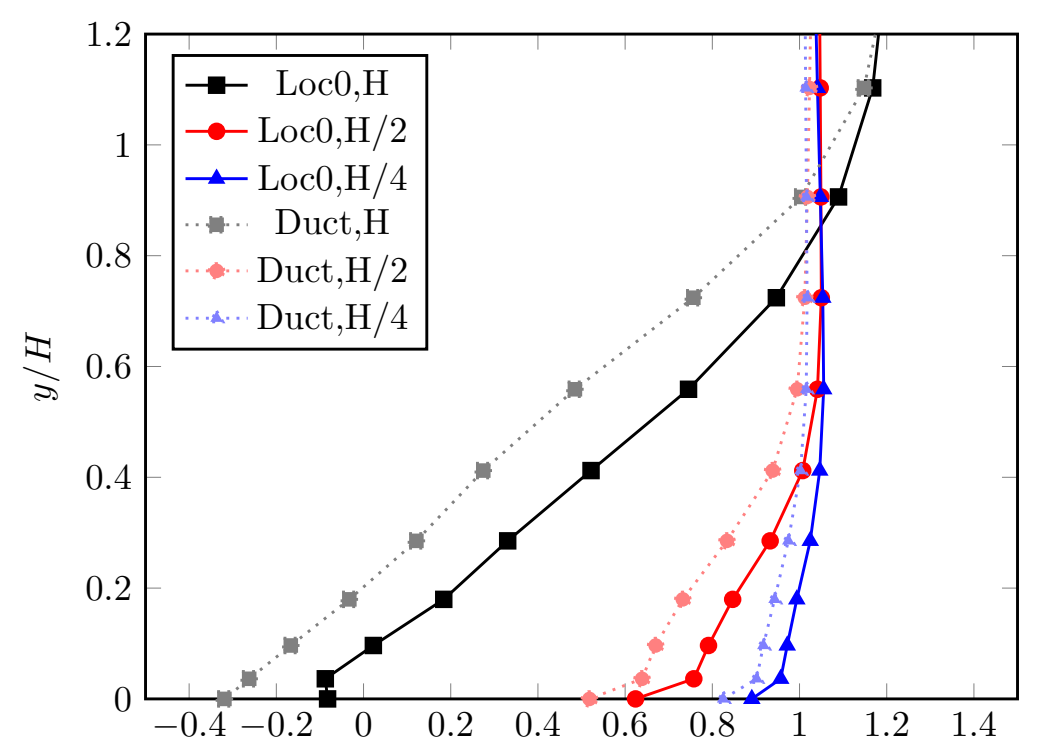

(a) Mass flux

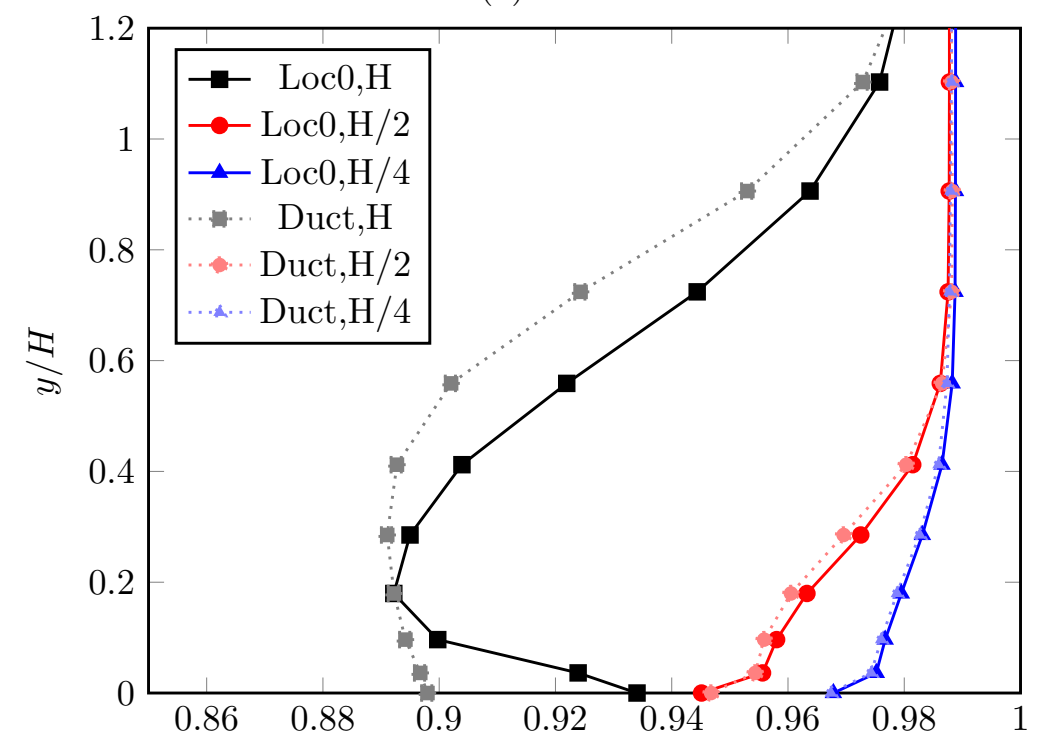

(b) Total pressure ratio

Fig. 18: Effect of different degrees of distortion on (a) mass flux and (b) total pressure ratio. 'Loc0' corresponds to the case with fan placed at $x=5.2 \mathrm{H}$ and 'Duct' corresponds to the case without fan. 